\title{
20 Specific corpora: Arithmetic, historiography, scientific poetry
}

The work of mathematicians of the third and the second centuries B.C. is perhaps the greatest - certainly it was the most permanent - achievement of Greek science.

Lloyd (1973: 51)

\$1 The data on the general corpus in the previous two chapters made it possible to demonstrate some differences between scientific Latin and other types of Latin, and it made it possible to differentiate between several different scientific styles. But, of course, the texts in that corpus are of a rather heterogeneous nature. For this reason, here some smaller corpora with texts from a single field but from different times are studied using similar methods, namely two scientific disciplines (arithmetic and historiography) and one genre (scientific poetry). Finding a scientific topic that is studied seriously and continuously across the two thousand years in Latin is surprisingly difficult: interests shifted, and some fields were abandoned or felt to be complete and not worthy of further study, while others were newly invented or rediscovered. A relatively easily definable and uncontroversial field with many theoretical contributions written in Latin is arithmetic. According to Dasypodius, $\Lambda \dot{\varepsilon} \xi ı \kappa o v$, p. 1r, it is defined thus:

Arithmetica es scientia, quae uim et naturam numerorum tradit: adfectiones etiam et accidentia per se eorundem explicat.

'Arithmetic is the science that studies the force and nature of numbers: it also explains relations and what happens between numbers.'

Table 16 shows the seventeen works on arithmetic used; they all treat numbers scientifically and are more than mere introductory compendia: thus, practical algorismi or Rechenbücher, commercial textbooks (De abaco), and the related field of computus were excluded. ${ }^{1}$ Commentaries were also excluded, as this genre is likely to behave in its own way, although there can be no doubt that it was important in the development of Latin arithmetic. Often, new insight was developed in the form of commentaries on older works, for instance in the case of Iacobus Faber Stapulensis' (ca. 1455-1536) commentary, Arithmetica decem, on Iordanus de Nemore's three-centuries-older treatise. In passing, it should also be mentioned that mathematics is a science in which on the one hand the vernacular was used

1 These criteria are not treated too rigidly before the twelfth century: if they were, we would be left with no Latin works at all. 
early on by Italian writers - such as Luca Paccioli (ca. 1447-1517), Niccolò Tartaglia (1499/1500-1557), or Rafael Bombelli (1526-1572) - but on the other hand important contributions were often written in Latin up to the nineteenth century (see chap. 14 §2). Of course, only Latin works are used in this chapter.

The topics relevant to the study of numbers changed over the centuries, as did the field's relation to geometry and algebra. Books VII-IX of Euclid's Elementa treat arithmetic in a geometric way and provided the foundation for practically all that followed in Latin arithmetic. While later on, arithmetic developed into its own independent field, for instance in Archimedes' Psammites, non-rational real numbers (such as $\pi$ ) were usually still dealt with in a geometric fashion, as there was no way of writing them as numbers. With the advent of differential calculus (Leibniz and Newton) the borders between arithmetic and geometry became even more blurred. Although there did exist Latin books on arithmetic before Boethius, they are unfortunately lost to us. For instance, we know that Varro's Disciplinae treated the artes liberales, ${ }^{2}$ and moreover that Apuleius wrote on mathematics as well. As the least explored times in Latin's development are after Antiquity, it may not be too inappropriate to begin this corpus as late as Martianus and Boethius and to focus more on the later phases of the language.

\$2 As many of these texts are of a rather specialist nature, little known to the general reader, their content and importance is at least summarised very briefly, illustrating some of the major themes that were under discussion during these different times. The texts can be read online in Corpus Corporum. General histories of mathematics paying at least some attention to historical and philological details are very rare. The usual approach is still that of a broad history of ideas, especially of ideas that were to last until today: to show how what we do today originated, at best mentioning some dead ends trodden in the past. ${ }^{3}$ For the important time of 1500 to 1740 , the monumental Latin-language work by Johann Christoph Heilbronner, Historia matheseos (Lipsiae, 1742), can still be useful. ${ }^{4}$

Arithmetic, as the scientific study of numbers and their properties, was developed by the Greeks hand-in-hand with numerology (the mystical properties of

2 Probably including arithmetica. See von Albrecht (1992-1994: 2:1243).

3 Smith (1923-1925) is still good reading, as well as Cantor (1880: 703-782), treating the Latin Middle Ages. For early prints of arithmetic texts, see Smith (1908). Gowers, Barrow-Green \& Leader (2008b) does contain lives of important mathematicians, but very few from before the seventeenth century.

4 On pp. 779-842, he lists authors on arithmetic in this timeframe. This book's other most lasting contribution may be the author's search for mathematical knowledge in the works of Aristotle (pp. 172-273). 
numbers). As already mentioned, the roots of the field can be seen in Euclid, who collected what was known in his day and whose work was so successful that it supplanted almost everything else, which is now lost for us. After Euclid, in the third and second centuries, there were great advances in the mathematical disciplines, achieved by men such as Archimedes, Eratosthenes, and Apollonius of Perga, which are, however, for the most part not well preserved. Rediscoveries of some of their works in the Renaissance were to change some sciences significantly. Instead of these highly technical, advanced works, the handbook by Nicomachos of Gerasa (fl. ca. AD 100), who was more interested in the philosophy of numbers than in strict proofs, was to become the foundation of later developments. Some later Greek authors such as Diophantus (third century AD) and Pappus (d. ca. AD 350) would, again, become important for the further development of the field when they were finally translated into Latin.

The earliest surviving Latin text on arithmetic is $§ \S 743-801$ of book VII of Martianus Capella's De nuptiis. ${ }^{5}$ Based on Euclid and Nicomachus, it explains the basics of number theory. The book was widely read in the Middle Ages. Boethius' De arithmetica is also based on Nicomachus' book; indeed, it is largely a translation of it. The treatment of this topic in Isidore's encyclopaedia is, again, based largely on Boethius and Cassiodorus (Institutiones II.4, too short to be included in the sample), and thus indirectly on Nicomachus. On the whole, it may be said that Latin Antiquity did not seem to add new knowledge to Euclid and Nicomachus. The same is largely true up to the twelfth century; ${ }^{6}$ Ps-Bede is rather a compilation for school use, possibly consisting of two parts that did not originally belong together. ${ }^{7}$ In the twelfth century, things change, as Euclid's Elementa is translated (see chap. $21 \S 2$ below) and is now directly accessible in full. The first surviving translation, by an anonymous Sicilian, is included in the sample (only the books treating arithmetic: VII-X). At roughly the same time, Arabic mathematics becomes available in Latin. Two texts about whose genesis and authorship very little is known are used (Anxiomata, Regule), except that they may have been written among the entourage of Adelard of Bath. ${ }^{8}$ Through the Arabs, Indian decimal numbers are introduced to Europe, greatly facilitating the handling of numbers in

5 The rest of book VII contains numerology and its literary framework. On author and work, see chap. $9 \S 5$ above.

6 As stressed above (chap. 9 §1): the Roman and early mediaeval periods can be seen as one period in the study of science in Latin.

7 Jones (1939: 48). This Ps-Bede text of practical character is edited by Folkerts (1972), who dates it to around 820. On Latin arithmetic before the translations, see Folkerts (2001).

8 Edited in Burnett (1996), besides Allard (1997: 212). 
arithmetic. ${ }^{9}$ Iordanus de Nemore's work in the thirteenth century is a first summarising reworking of the new knowledge and would be very successful for a long time. Iohannes de Muris is, again, largely based on Boethius and was used as a relatively elementary schoolbook. The two works from the fourteenth century (Albert of Saxony and Nicolaus Oresmius) use arithmetic for physical problems, but both do so at a very theoretical level and can be seen as precursors of mechanics in the Scientific Revolution. The Austrian astronomer Iohannes de Gamundia (of Gmunden) treats arcs and angles numerically in his De sinubus et chordis. Franciscus Maurolycus, an Italian with Greek roots, was also a student of Greek texts and learned from Diophantus; ${ }^{10}$ his work on arithmetic was meant to go beyond Euclid, Nicomachus, and Iordanus (I, prol., 1575 edition, p. 1). With Franciscus Vieta (and John Wallis), a new interest in the infinite becomes apparent, culminating in Leibniz's and Newton's calculus. The text by Leibniz is one of the foundational works of infinitesimal calculus. ${ }^{11}$ This is the first work that looks like a contemporary mathematics paper. It is full of formulas and very much to the point. The author does not care about rhetorical language at all; he makes up unusual words such as infinitangulum ('apeirogon'; 470) without any apologies. Leonard Euler's short study also follows these lines, ${ }^{12}$ whereas Gauß's work combines number theory and algebra, reworking much of the advances of the past century and introducing much novelty of his own. The book is a classic in the field. It also reads like a modern mathematics textbook. Gauß's works are among the last fundamentally important works in the field first published in Latin. ${ }^{13}$

Unfortunately, few of these rather technical texts were available in digital form. Were it not for Busard's tireless editing, few of the mediaeval ones would even be available in printed editions. Since I had to digitise many of the texts in this corpus myself, the texts used tend to be relatively short excerpts, which may render the results less certain.

\$3 Comparative tables provide basic information about the authors, works, and editions (table 16-17) used in this chapter. For the post-classical vocabulary (as for the general corpus above), the 1,000 words after the first 1,000 words were

\footnotetext{
9 Ambrosetti (2008: esp. chap. 10) studies the Arabic influence in late mediaeval and early modern mathematics.

10 His many works are currently being edited at the University of Pisa.

11 On its development, see Spalt (2015).

12 His works can be found online at http://eulerarchive.maa.org/index.html.

13 Especially his Disquisitiones generales circa superficies curvas (Göttingen: typis Dieterichianis, 1828); there are some later important but short works, such as Giuseppe Peano's Arithmetices principia (Turin: Bocca, 1889; https://archive.org/details/arithmeticesprio0peangoog).
} 
studied and compared to surviving literature before AD 200 as contained in Corpus Corporum. The lists are printed in appendix 2. Somewhat surprisingly, the mediaeval authors hardly use non-classical words, whereas the late antique and the early modern ones do so profusely, especially Martianus Capella, Maurolycus, and Leibniz. The arithmetic texts contain in total 166 [574] such lemmata [occurrences]. They include 92 [381] ADJ, 3 [6] ADV, 56 [148] N, 12 [17] V, and 3 [22] PRON. Here, ADJ are more common than N. The structure of the works is strongly influenced by Euclid as soon as his text becomes available. Early modern authors tend to follow the same structure still in use today: definitions are followed by propositions and their proofs. The use of formulas also becomes more common with time.

Table 16: The seventeen arithmetic texts used in this corpus.

\begin{tabular}{|c|c|c|c|c|c|c|c|}
\hline Author and work ${ }^{14}$ & $\begin{array}{l}\text { Life dates or } \\
\text { date of } \\
\text { publication }\end{array}$ & $\begin{array}{l}\text { Topic of the } \\
\text { work }\end{array}$ & $\begin{array}{l}\text { No. of } \\
\text { words, } \\
\text { avg word } \\
\text { length }\end{array}$ & $\begin{array}{l}\text { Post-classical } \\
\text { words, types/ } \\
\text { tokens }^{16}\end{array}$ & $\begin{array}{l}\text { Brief } \\
\text { description } \\
\text { of style }\end{array}$ & $\begin{array}{l}\text { Knew } \\
\text { Greek? }\end{array}$ & $\begin{array}{l}\text { Diagrams, } \\
\text { formulas, } \\
\text { graphics, } \\
\text { proofs? }\end{array}$ \\
\hline $\begin{array}{l}\text { Martianus Capella, Du } \\
\text { nuptiis VII, } \\
\text { ed. Willis (1983) }\end{array}$ & 5th century & $\begin{array}{l}\text { introduction } \\
\text { based on } \\
\text { Nicomachus }\end{array}$ & $\begin{array}{r}10 \\
5.64\end{array}$ & $\begin{array}{l}17 \\
43\end{array}$ & discursive & $\mathrm{Y} ?$ & N \\
\hline $\begin{array}{l}\text { Boethius, De } \\
\text { arithmetica } \\
\text { PL } 63 \text { (1863) }\end{array}$ & $\begin{array}{l}\text { ca. 480- } \\
524\end{array}$ & $\begin{array}{l}\text { introduction } \\
\text { based on } \\
\text { Nicomachus }\end{array}$ & $\begin{array}{r}30 \\
6.31\end{array}$ & \begin{tabular}{r|}
9 \\
12
\end{tabular} & $\begin{array}{l}\text { discursive } \\
\text { with conclu- } \\
\text { siones and } \\
\text { probatur }\end{array}$ & Y & N \\
\hline $\begin{array}{l}\text { Isidore, Etymologiae } \\
\text { III.2-9, } \\
\text { ed. Lindsay (1911) }\end{array}$ & $\begin{array}{l}\text { ca. } 560- \\
636\end{array}$ & $\begin{array}{l}\text { introduction } \\
\text { based on } \\
\text { Nicomachus }\end{array}$ & $\begin{array}{r}2 \\
6.05\end{array}$ & $\begin{array}{l}18 \\
47\end{array}$ & discursive & $N$ & $\mathrm{~N}$ \\
\hline $\begin{array}{l}\text { Ps-Bede, De } \\
\text { arithmeticis propositio- } \\
\text { nibus PL } 90 \text { (1904) }\end{array}$ & ca. 820 & schoolbook & $\begin{array}{r}5 \\
6.05\end{array}$ & $\begin{array}{l}5 \\
8\end{array}$ & discursive & $N$ & $\mathrm{~N}$ \\
\hline $\begin{array}{l}\text { Anxiomata artis } \\
\text { arithmeticae, } \\
\text { ed. Burnett (1996) }\end{array}$ & $\begin{array}{l}\text { mid-12th } \\
\text { century }\end{array}$ & $\begin{array}{l}\text { definitions } \\
\text { and basic } \\
\text { rules }\end{array}$ & $\begin{array}{r}2 \\
4.93\end{array}$ & $\begin{array}{l}15 \\
43\end{array}$ & exercises & $N$ & $N$ \\
\hline $\begin{array}{l}\text { Anonymus Toletanus, } \\
\text { Regule, } \\
\text { ed. Burnett (2010) }\end{array}$ & ca. 1170 & $\begin{array}{l}\text { basic rules } \\
\text { and questions }\end{array}$ & $\begin{array}{r}10 \\
5.95\end{array}$ & $\begin{array}{r}4 \\
13\end{array}$ & discursive & $N$ & D \\
\hline
\end{tabular}

14 Red means less than the average minus 1 stdev, green more than the average plus 1 stdev.

15 In thousands. The shortest texts are around 2,200 words; most are much longer. The average word length for these texts is $5.97 \pm 0.52$, almost identical to the benchmark: $5.95 \pm 0.32$.

16 The average numbers for these texts are $12.2 \pm 6.9$ and $33.4 \pm 24$. 3 . The numbers are not comparable to those in chap. 18 as a smaller sample was used to obtain them. 
Table 16: (continued)

\begin{tabular}{|c|c|c|c|c|c|c|c|}
\hline $\begin{array}{l}\text { Anonymus Siculus, } \\
\text { Elementa translation } \\
\text { (books VII-X), ed. } \\
\text { Busard (1987) }\end{array}$ & ca. 1175 & $\begin{array}{l}\text { translation of } \\
\text { Euclid }\end{array}$ & $\begin{array}{r}41 \\
4.80\end{array}$ & $\begin{array}{r}2 \\
16\end{array}$ & $\begin{array}{l}\text { definitiones, } \\
\text { propositio- } \\
\text { nes, proofs } \\
\text { (geometric) }\end{array}$ & Y & $\begin{array}{l}\mathrm{D} \\
\mathrm{P}\end{array}$ \\
\hline $\begin{array}{l}\text { Iordanus de Nemore, De } \\
\text { elementis arithmetice } \\
\text { artis, ed. Busard (1991) }\end{array}$ & fl. ca. 1230 & $\begin{array}{l}\text { compendium } \\
\text { including } \\
\text { proofs }\end{array}$ & $\begin{array}{r}45 \\
6.14\end{array}$ & $\begin{array}{l}2 \\
6\end{array}$ & $\begin{array}{l}\text { definitiones, } \\
\text { propositio- } \\
\text { nes, proofs } \\
\text { (geometric) }\end{array}$ & $\mathrm{N}$ ? & $P$ \\
\hline $\begin{array}{l}\text { Iohannes de Muris, } \\
\text { Arithmetica speculati- } \\
\text { va, ed. Busard (1971) }\end{array}$ & 1343 & $\begin{array}{l}\text { problems, of- } \\
\text { ten about } \\
\text { proportions }\end{array}$ & $\begin{array}{r}7 \\
6.25\end{array}$ & $\begin{array}{r}9 \\
27\end{array}$ & $\begin{array}{l}\text { discursive } \\
\text { with defini- } \\
\text { tions }\end{array}$ & $\mathrm{N} ?$ & $\begin{array}{l}\mathrm{D} \\
\mathrm{F}\end{array}$ \\
\hline $\begin{array}{l}\text { Albert of Saxony, De } \\
\text { proportionum, } \\
\text { ed. Busard (1971) }\end{array}$ & $\begin{array}{l}\text { ca. } 1320- \\
1390\end{array}$ & $\begin{array}{l}\text { proportions } \\
\text { and their use } \\
\text { in physics }\end{array}$ & $\begin{array}{r}7 \\
6.11\end{array}$ & $\begin{array}{c}17 \\
86\end{array}$ & $\begin{array}{l}\text { discursive } \\
\text { with conclu- } \\
\text { siones and } \\
\text { probatur }\end{array}$ & $\mathrm{N}$ ? & $P$ \\
\hline $\begin{array}{l}\text { Nicolaus Oresmius, De } \\
\text { proportionibus propor- } \\
\text { tionum, ed. Grant } \\
(1966)\end{array}$ & $\begin{array}{l}\text { ca. } 1320- \\
1382\end{array}$ & $\begin{array}{l}\text { proportions } \\
\text { and their use } \\
\text { in physics }\end{array}$ & $\begin{array}{r}20 \\
5.82\end{array}$ & $\begin{array}{r}15 \\
39\end{array}$ & $\begin{array}{l}\text { discursive } \\
\text { with conclu- } \\
\text { siones and } \\
\text { probatur }\end{array}$ & $\mathrm{N}$ ? & $\begin{array}{l}P \\
F\end{array}$ \\
\hline $\begin{array}{l}\text { Iohannes de Gamundia, } \\
\text { De sinibus et chordis, } \\
\text { ed. Busard (1971) }\end{array}$ & 1437 & trigonometry & $\begin{array}{r}18 \\
5.76\end{array}$ & $\begin{array}{r}7 \\
23\end{array}$ & $\begin{array}{l}\text { discursive } \\
\text { with proofs }\end{array}$ & $Y ?$ & D \\
\hline $\begin{array}{l}\text { Franciscus Maurolycus, } \\
\text { Arithmeticorum libri } \\
\text { duo, ed. Pasquotto } \\
\text { (2017) }\end{array}$ & 1575 & number theory & $\begin{array}{r}27 \\
6.78\end{array}$ & $\begin{array}{l}26 \\
91\end{array}$ & $\begin{array}{l}\text { definitiones, } \\
\text { propositio- } \\
\text { nes, proofs }\end{array}$ & $\mathrm{Y}$ & $\begin{array}{l}P \\
F\end{array}$ \\
\hline $\begin{array}{l}\text { Franciscus Vieta, In } \\
\text { artem analyticem } \\
\text { isagoge (Lugduni } \\
\text { Batavorum, 1646) }\end{array}$ & 1591 & $\begin{array}{l}\text { analytic geo- } \\
\text { metry }\end{array}$ & $\begin{array}{r}5 \\
6.60\end{array}$ & $\begin{array}{r}11 \\
29\end{array}$ & $\begin{array}{l}\text { discursive } \\
\text { with proofs }\end{array}$ & $\mathrm{Y}$ & $\begin{array}{l}P \\
F\end{array}$ \\
\hline $\begin{array}{l}\text { Gottfried Wilhelm } \\
\text { Leibniz, Nova metho- } \\
\text { dus pro maximis et } \\
\text { minimis (Lipsiae, 1684) }\end{array}$ & 1684 & calculus & $\begin{array}{r}2 \\
5.72\end{array}$ & $\begin{array}{l}21 \\
37\end{array}$ & $\begin{array}{l}\text { definitiones, } \\
\text { propositio- } \\
\text { nes, proofs }\end{array}$ & $\mathrm{Y}$ & $\begin{array}{l}P \\
F\end{array}$ \\
\hline $\begin{array}{l}\text { Leonhard Euler, } \\
\text { Introductio in analysin } \\
\text { infinitorum (Petropolis, } \\
1740 \text { ) }\end{array}$ & 1748 & calculus & $\begin{array}{r}3 \\
6.23\end{array}$ & $\begin{array}{l}12 \\
19\end{array}$ & $\begin{array}{l}\text { definitiones, } \\
\text { propositio- } \\
\text { nes, proofs }\end{array}$ & $\mathrm{Y}$ & $\begin{array}{l}P \\
F\end{array}$ \\
\hline $\begin{array}{l}\text { Carl Friedrich Gauß, } \\
\text { Disquisitiones arithme- } \\
\text { ticae (sections } \\
\text { 1-2) (Leipzig, 1801) }\end{array}$ & 1801 & $\begin{array}{l}\text { systematic al- } \\
\text { gebra and } \\
\text { arithmetic }\end{array}$ & $\begin{array}{r}8 \\
6.41\end{array}$ & $\begin{array}{l}17 \\
28\end{array}$ & $\begin{array}{l}\text { definitiones, } \\
\text { propositio- } \\
\text { nes, proofs }\end{array}$ & $\mathrm{Y}$ & $\begin{array}{l}P \\
F\end{array}$ \\
\hline
\end{tabular}




\begin{tabular}{|c|c|c|c|c|c|c|c|c|c|c|c|c|}
\hline 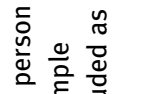 & Entropy & * & 刑 & * & * & * & $\stackrel{ก}{\wedge}$ & సี่| & 节 & * & * & ำ: \\
\hline 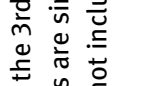 & ABL ABS & إْ & $\underset{f}{\vec{g}}$ & 잉 & సू & वे. & $\begin{array}{l}0 \\
\\
i \\
i\end{array}$ & $\begin{array}{l} \\
\\
0\end{array}$ & 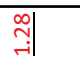 & $\stackrel{\sim}{\alpha}$ & $\underset{\rightarrow}{m}$ & 엠| \\
\hline 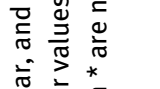 & Modifiers & ๙ึ้ & $\begin{array}{l}\infty \\
\vdots \\
m\end{array}$ & \begin{tabular}{|l|l} 
\\
$\substack{0 \\
+}$ \\
+
\end{tabular} & $\underset{\sim}{n}$ & $\underset{\sim}{\stackrel{N}{\sim}}$ & $\begin{array}{l}\infty \\
\stackrel{\infty}{i} \\
\dot{i}\end{array}$ & $\begin{array}{l} \\
0 \\
\vdots \\
\vdots\end{array} \mid$ & 각 & $\underset{i}{\stackrel{t}{i}}$ & 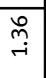 & $\begin{array}{l}m \\
\substack{\infty \\
\rightarrow} \\
\rightarrow+\end{array}$ \\
\hline 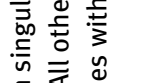 & N-ADJ & 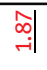 & 잇 & 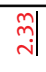 & बे & 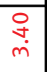 & $\begin{array}{l}\infty \\
\stackrel{m}{m}\end{array}$ & $\begin{array}{l}\infty \\
\stackrel{0}{m} \\
\dot{m}\end{array}$ & $\begin{array}{l}n \\
\dot{n} \\
\dot{m}\end{array}$ & $\begin{array}{l}\dot{y} \\
\dot{m}\end{array}$ & 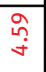 & $\begin{array}{l}\text { ? } \\
\infty \\
\infty\end{array}$ \\
\hline $\begin{array}{l}\dot{\overline{0}} \\
\dot{0} \\
\frac{0}{0}\end{array}$ & ADJ-SUF & 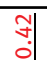 & ? & $\begin{array}{l}\overrightarrow{-} \\
\overrightarrow{+} \\
-i\end{array}$ & \begin{tabular}{l}
$\infty$ \\
\multirow{0}{0}{} \\
0
\end{tabular} & 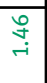 & $\underset{\sim}{\stackrel{n}{r}}$ & mैa & $\stackrel{\substack{n \\
\rightarrow}}{\rightarrow}$ & 喿| & 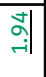 & 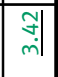 \\
\hline . & 3rd PAS & 춴 & 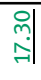 & 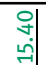 & $\begin{array}{l}\infty \\
\stackrel{\infty}{0} \\
\dot{0}\end{array}$ & 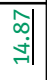 & 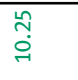 & 害 & 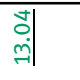 & 원 & $\begin{array}{ll}0 \\
2 \\
0 \\
0\end{array}$ & 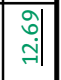 \\
\hline$\frac{0}{\frac{0}{0}}$ & 1st SG & $\stackrel{\substack{h \\
\sim}}{\rightarrow}$ & $\underset{+}{\stackrel{f}{r}}$ & 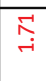 & $\vec{i}$ & $\begin{array}{l}\hat{N} \\
0 \\
0\end{array}$ & $\begin{array}{l}\hat{n} \\
\hat{o}\end{array}$ & $\begin{array}{l}\vec{b} \\
\dot{m}\end{array}$ & $\underset{i}{\text { O }}$ & $\begin{array}{l}\infty \\
\stackrel{\infty}{0} \\
0\end{array}$ & \begin{tabular}{l} 
\\
\multirow{U}{*}{} \\
i
\end{tabular} & $\vec{a}$ \\
\hline $\overrightarrow{0}$ & PRON:POSS & गֶ̃. & 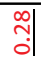 & \begin{tabular}{|l|}
\multicolumn{2}{c}{$\mid$} \\
0 \\
0
\end{tabular} & ㅇํㅇ & ôn. & $\vec{m} \dot{0} \mid$ & 家 & : & 告| & : & 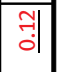 \\
\hline Oั & CONJ:S & $\stackrel{\infty}{\sim}$ & $\tilde{m}$ & \begin{tabular}{|l|}
$N$ \\
$N$ \\
$m$
\end{tabular} & $\stackrel{\hat{n}}{i}$ & 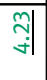 & $\stackrel{\circ}{\circ}$ & $\begin{array}{l}\infty \\
\infty \\
i \\
i\end{array}$ & 국 & $\underset{m}{\tilde{m}}$ & $\begin{array}{ll}\stackrel{2}{\infty} \\
\stackrel{\sim}{ }\end{array}$ & à \\
\hline 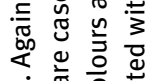 & REL & $\begin{array}{l}\stackrel{0}{n} \\
\dot{m}\end{array}$ & : & $\begin{array}{l}N \\
m \\
m\end{array}$ & $\underset{m}{N}$ & $\begin{array}{l}\infty \\
\stackrel{\infty}{i} \\
\dot{N}\end{array}$ & $\stackrel{\hat{n}}{m}$ & $\begin{array}{l}\stackrel{0}{0} \\
\dot{m}\end{array}$ & $\underset{m}{\stackrel{m}{m}}$ & $\hat{\circ}$ & $\stackrel{\hat{n}}{m}$ & $\underset{\sim}{\stackrel{N}{m}}$ \\
\hline$\stackrel{0}{\equiv}$ & ESSE & 氛 & 密 & 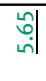 & $\stackrel{\tilde{m}}{\dot{\sim}}$ & จุำ & $\begin{array}{c}M \\
\infty \\
\dot{f}\end{array}$ & श्र & ָָ๊ & ?ं। & 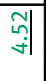 & \begin{tabular}{|c|c|}
$\hat{0}$ \\
$\infty$ \\
$\infty$
\end{tabular} \\
\hline 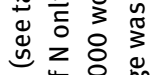 & $\overline{A B L / D A T}$ & 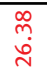 & $\begin{array}{l}\vec{m} \\
\text { ஸे }\end{array}$ & $\begin{array}{l}0 \\
\text { ì } \\
\text { in }\end{array}$ & $\begin{array}{l}n \hat{n} \\
\stackrel{i}{N}\end{array}$ & 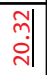 & 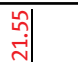 & $\begin{array}{l}\text { I } \\
\infty \\
0 \\
0\end{array}$ & 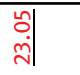 & $\begin{array}{l}m \\
m \\
\text { à }\end{array}$ & 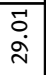 & \begin{tabular}{|l|}
$m$ \\
$m$ \\
$\dot{n}$
\end{tabular} \\
\hline 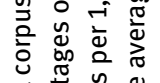 & $\mathrm{ACC}$ & \begin{tabular}{l} 
mad \\
\multirow{2}{*}{$\mid$}
\end{tabular} & $\begin{array}{l}0 \\
0 \\
\vdots \\
\cdots\end{array} \mid$ & \begin{tabular}{|l|}
\multirow{2}{*}{} \\
ते
\end{tabular} & वี & 害| & ָั & $\begin{array}{l}\overrightarrow{1} \\
\stackrel{1}{0} \\
\text { m. }\end{array}$ & $\begin{array}{l}\text { a } \\
\text { : } \\
\text { i }\end{array}$ & वै| & $\begin{array}{l}\text { N } \\
\stackrel{N}{0} \\
\text { N }\end{array}$ & 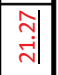 \\
\hline 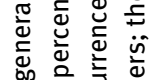 & GEN & $\begin{array}{l}\hat{m} \\
\text { aे }\end{array}$ & t & 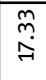 & $\mid \begin{array}{c}m \\
\stackrel{m}{*}\end{array}$ & 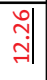 & ঐి & $\begin{array}{l}\text { oे } \\
\text { जे }\end{array}$ & 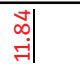 & 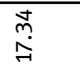 & 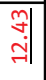 & $\begin{array}{l}\text { न } \\
\text { 음 }\end{array}$ \\
\hline 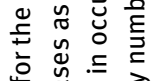 & NOM & \begin{tabular}{l|l} 
\\
$\infty$ \\
$\infty$ \\
$\vdots$
\end{tabular} & 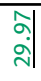 & 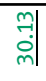 & $\stackrel{\underset{7}{+}}{\stackrel{\sim}{N}}$ & $\stackrel{\substack{n \\
m}}{m}$ & $\begin{array}{l}\overrightarrow{1} \\
\dot{m} \\
\text { ind }\end{array}$ & 凋| & 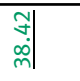 & $\begin{array}{l}\text { ợ } \\
\text { ^ै। }\end{array}$ & 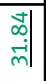 & 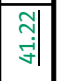 \\
\hline$\stackrel{0}{ \pm}$ & PTC & ले & 9 & \begin{tabular}{|l|l}
$\infty$ \\
$\stackrel{n}{\sim}$ \\
\end{tabular} & $\begin{array}{l}\hat{a} \\
\text { فे }\end{array}$ & \begin{tabular}{l} 
\\
\multirow{2}{*}{} \\
\end{tabular} & $\vec{m}$ & $\begin{array}{l}\stackrel{0}{7} \\
\stackrel{7}{7}\end{array}$ & $\stackrel{\stackrel{n}{\sim}}{\stackrel{A}{A}}$ & 究 & $\begin{array}{l}\overrightarrow{0} \\
\dot{a} \\
\dot{a}\end{array}$ & 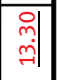 \\
\hline 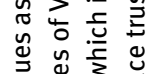 & INF & 국 & 声 & न्नु & $\stackrel{\sim}{\tilde{\omega}}$ & 要 & $\begin{array}{l}\overrightarrow{7} \\
\text { in }\end{array}$ & | & वे & 忒 & 望 & $\stackrel{\infty}{\Lambda}$ \\
\hline$\stackrel{0}{\frac{\pi}{2}}$ & SUB & $\begin{array}{l}\hat{n} \\
\dot{y}\end{array}$ & 1 & 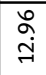 & 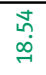 & 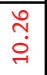 & $\begin{array}{l}\stackrel{\omega}{m} \\
\stackrel{m}{n}\end{array}$ & $\begin{array}{l}\hat{\sim} \\
\ddot{\sim}\end{array}$ & \begin{tabular}{l|} 
\\
일. \\
|
\end{tabular} & 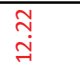 & $\begin{array}{l}\hat{A} \\
\Leftrightarrow\end{array}$ & न्न \\
\hline 品홍호 & IND & 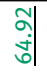 & $\begin{array}{ll} & 0 \\
\infty \\
0 \\
0\end{array}$ & 월 & $\begin{array}{l}\text { ọ } \\
\text { in }\end{array}$ & 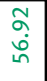 & $\begin{array}{l}\hat{n} \\
\hat{m} \\
\stackrel{\omega}{n}\end{array}$ & 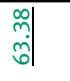 & $\begin{array}{l}\text { aे } \\
\text { o }\end{array}$ & \begin{tabular}{l|}
0 \\
0 \\
f.
\end{tabular} & $\begin{array}{l}\text { ने } \\
\text { 出 } \\
\end{array}$ & $\begin{array}{l}m \\
\\
\end{array}$ \\
\hline 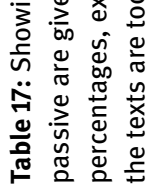 & $\bar{x}$ & 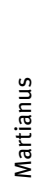 & . & $\begin{array}{l}\frac{0}{0} \\
\frac{0}{n}\end{array}$ & $\begin{array}{l}\stackrel{0}{\bar{d}} \\
\dot{\infty} \\
\dot{\sim}\end{array}$ & 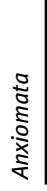 & 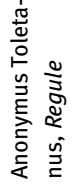 & 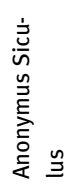 & 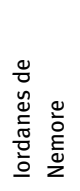 & 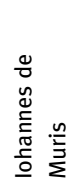 & 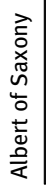 & 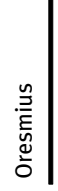 \\
\hline
\end{tabular}




\begin{tabular}{|c|c|c|c|c|c|c|c|c|c|c|c|c|}
\hline Entropy & 윗 & 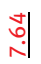 & $*$ & * & * & * & 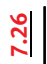 & : & $\underset{\infty}{\infty}$ & $\left|\begin{array}{l}\sigma \\
\alpha\end{array}\right|$ & $\stackrel{2}{2}$ & 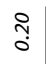 \\
\hline ABL ABS & 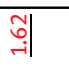 & $\stackrel{\sim}{\stackrel{n}{m}}$ & $\begin{array}{l}\stackrel{n}{\circ} \\
\dot{j}\end{array}$ & $\underset{m}{\stackrel{m}{m}}$ & \begin{tabular}{l|l|} 
f \\
fil
\end{tabular} & $\begin{array}{l}\stackrel{\circ}{\circ} \\
\text { in }\end{array}$ & 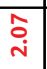 & $\underset{i}{\infty}$ & $\begin{array}{l}\mathbf{\infty} \\
\stackrel{\sim}{0} \\
\text { in }\end{array}$ & 高| & $\stackrel{m}{\dot{m}}$ & $\begin{array}{l}\overrightarrow{7} \\
0\end{array}$ \\
\hline Modifiers & $\begin{array}{l}\omega_{\infty} \\
\stackrel{\sim}{+}\end{array}$ & 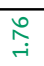 & $\underset{i}{\stackrel{J}{i}}$ & $\underset{\sim}{\stackrel{\sim}{r}}$ & 畓| & 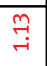 & 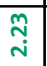 & 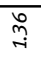 & 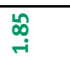 & \begin{tabular}{|l|}
$\overrightarrow{0}$ \\
$\vdots$ \\
0
\end{tabular} & ঙ̣̊ & 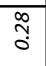 \\
\hline N-ADJ & $\begin{array}{l}\tilde{N} \\
\dot{m}\end{array}$ & 字 & సู| & $\underset{\dot{\sigma}}{\hat{\sigma}}$ & \begin{tabular}{|l|}
$\infty$ \\
0 \\
0 \\
$\dot{n}$
\end{tabular} & $\underset{\sim}{\stackrel{\sim}{\sim}}$ & $\begin{array}{l}\stackrel{8}{0} \\
\dot{y}\end{array}$ & $\stackrel{\grave{i}}{i}$ & $\begin{array}{l}\stackrel{0}{\circ} \\
\dot{j}\end{array}$ & $\mid \begin{array}{c}\widehat{o} \\
0 \\
0\end{array}$ & 궇 & $\begin{array}{l}\bar{n} \\
0\end{array}$ \\
\hline ADJ-SUF & $\begin{array}{l}\mid \hat{W} \\
\text { in }\end{array}$ & ?: & 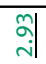 & $\begin{array}{l}\hat{0} \\
+ \\
\dot{\gamma}\end{array}$ & $\begin{array}{l}\infty \\
\\
\vdots \\
0\end{array}$ & 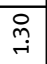 & 일 & $\underset{7}{\stackrel{9}{7}}$ & 齐 & 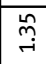 & $\underset{\sim}{\sim}$ & $\stackrel{\infty}{\stackrel{\infty}{0}}$ \\
\hline 3rd PAS & |: & $\begin{array}{l}\text { ơ } \\
\text { a }\end{array}$ & \begin{tabular}{l}
$m$ \\
$m$ \\
\hdashline
\end{tabular} & $\begin{array}{l}0 \\
\text { m. } \\
\text { o. }\end{array}$ & \begin{tabular}{|l|l|}
0 \\
0 \\
0 \\
0
\end{tabular} & 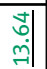 & 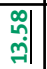 & $\underset{\forall}{\mathbb{V}}$ & 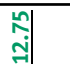 & $\mid$\begin{tabular}{c}
$\hat{n}$ \\
\multirow{j}{*}{$\mid$}
\end{tabular} & ন্: & ồ \\
\hline 1st SG & $\underset{\sim}{\sim}$ & 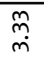 & $\stackrel{\sim}{\sim}_{+}^{\infty}$ & $\begin{array}{l}\overrightarrow{+} \\
\vec{r}\end{array}$ & \begin{tabular}{l|}
$\stackrel{N}{M}$ \\
$\dot{m}$
\end{tabular} & $\begin{array}{c}\alpha \\
\alpha \\
o\end{array}$ & 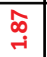 & $\stackrel{\vec{H}}{\vec{H}}$ & 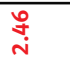 & $\underset{\sim}{\tilde{n}}$ & $\underset{j}{J}$ & $\underset{-1}{+}$ \\
\hline PRON:POSS & |l| & 苟| & ㄱํㅇ & : & 跑 & ?े| & \begin{tabular}{|l|}
$\vec{m}$ \\
○ी
\end{tabular} & חֶ. & 咅| & 党 & ఝొ & $\begin{array}{l}\hat{n} \\
\hat{0} \\
0\end{array}$ \\
\hline CONJ:S & 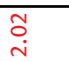 & 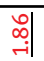 & 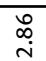 & $\begin{array}{l}\text { aे } \\
\text { min }\end{array}$ & 웜 & $\underset{m}{\stackrel{H}{m}}$ & $\underset{m}{\stackrel{9}{m}}$ & 帘 & ষे & 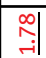 & నু & $\bar{~}$ \\
\hline REL & $\overrightarrow{\mathrm{m}}$ & 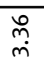 & $\stackrel{\text { 움 }}{\text { r }}$ & 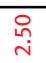 & \begin{tabular}{|l|}
$\vec{\infty}$ \\
$\dot{m}$
\end{tabular} & $\begin{array}{l}\infty \\
\stackrel{\infty}{\sim} \\
\dot{\sim}\end{array}$ & $\stackrel{\hat{m}}{m}$ & 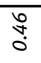 & $\stackrel{\Xi}{\mathrm{m}}$ & 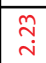 & $\stackrel{\substack{\hat{m} \\
m}}{n}$ & ?̂. \\
\hline ESSE & \begin{tabular}{|l|}
$\vec{b}$ \\
$\dot{0}$
\end{tabular} & \begin{tabular}{|c|c|}
0 \\
$\dot{y}$ \\
\hdashline
\end{tabular} & 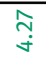 & 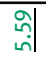 & \begin{tabular}{|l|l|} 
\\
$\vdots$ \\
\multirow{y}{*}{$\mid$}
\end{tabular} & 矛 & 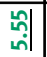 & $\underset{\sim}{\tilde{r}}$ & 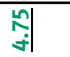 & ঐ్ & $\underset{m}{\mathbb{N}}$ & \begin{tabular}{l}
\multirow{2}{*}{} \\
\hdashline
\end{tabular} \\
\hline$\overline{\mathrm{ABL} / \mathrm{DAT}}$ & 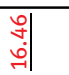 & $\begin{array}{l}\overrightarrow{7} \\
\text { 究 }\end{array}$ & 离 & 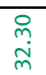 & \begin{tabular}{l|} 
\\
$\vdots$ \\
$\vdots$
\end{tabular} & 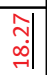 & 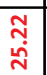 & ऊे & $\begin{array}{l}\stackrel{m}{a} \\
\text { iे }\end{array}$ & \begin{tabular}{|l|} 
\\
$\stackrel{\infty}{+}$ \\
$\stackrel{0}{m}$
\end{tabular} & ホ̊. & $\widehat{\hat{i}}$ \\
\hline $\mathrm{ACC}$ & $\begin{array}{l}\infty \\
\stackrel{\infty}{\sim} \\
\text { స. }\end{array}$ & $\begin{array}{l}\text { ले| } \\
\text { ने| }\end{array}$ & 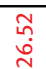 & 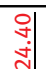 & \begin{tabular}{|c|}
$\infty$ \\
$\infty$ \\
$\infty$ \\
$\infty$ \\
$\sim$
\end{tabular} & $\begin{array}{l}\stackrel{?}{\sim} \\
\stackrel{\sim}{\sim}\end{array}$ & 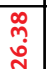 & 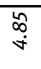 & $\begin{array}{l}\text { \& } \\
\text { ஸે } \\
\end{array}$ & $\begin{array}{l}\mid \\
\mathrm{n} \\
\mathrm{m}\end{array}$ & $\underset{\sim}{\infty}$ & $\underset{H}{Z}$ \\
\hline GEN & $\begin{array}{l}\tilde{N} \\
\stackrel{\sim}{\mathbf{N}}\end{array}$ & $\begin{array}{l}\overrightarrow{1} \\
\dot{d} \\
\dot{d}\end{array}$ & 点 & 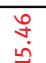 & \begin{tabular}{l|}
$\stackrel{n}{m}$ \\
$m$ \\
$m$
\end{tabular} & $\begin{array}{l}\tilde{n} \\
\dot{v}\end{array}$ & 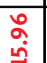 & $\begin{array}{l}\forall \\
\text { m }\end{array}$ & 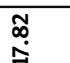 & i̊n. & $\underset{\infty}{\infty}$ & $\stackrel{n}{n}$ \\
\hline NOM & $\begin{array}{l}\stackrel{m}{N} \\
\vec{m}\end{array}$ & $\begin{array}{l}0 \\
0 \\
\dot{m}\end{array}$ & $\begin{array}{l}\hat{b} \\
\dot{j}\end{array}$ & 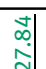 & श्: & $\begin{array}{l}\text { 위 } \\
\infty\end{array}$ & 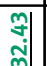 & $\begin{array}{l} \\
\infty \\
\forall \\
\forall\end{array}$ & సั้ & $\begin{array}{l}\vec{m} \\
\dot{\sim} \\
\dot{\sim}\end{array}$ & 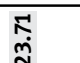 & $\underset{7}{\stackrel{9}{7}}$ \\
\hline PTC & $\begin{array}{l}\text { oे } \\
\vec{m}\end{array}$ & गें & 旄 & $\begin{array}{l}\mathbf{N} \\
\vdots \\
\vdots\end{array}$ & \begin{tabular}{|l|}
$\infty$ \\
$\stackrel{N}{\sim}$ \\
$\sim$
\end{tabular} & \begin{tabular}{l}
$\mathbb{N}$ \\
\multirow{6}{0}{} \\
\end{tabular} & 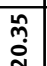 & فे & 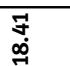 & \begin{tabular}{l} 
\\
\multirow{j}{*}{}
\end{tabular} & 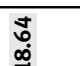 & הิ \\
\hline INF & 일. & 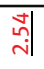 & $\begin{array}{l}9 \\
0 \\
0\end{array}$ & $\begin{array}{l}\vec{N} \\
\vec{G}\end{array}$ & $\begin{array}{l}\stackrel{2}{2} \\
a\end{array}$ & \begin{tabular}{l}
$\mathbb{N}$ \\
\multirow{6}{0}{}
\end{tabular} & $\begin{array}{l}8 \\
\text { in }\end{array}$ & $\begin{array}{l}\tilde{\sigma} \\
\dot{m}\end{array}$ & 옥 & $\begin{array}{l}\infty \\
\stackrel{\infty}{0} \\
\stackrel{9}{\circ}\end{array}$ & $\underset{\text { ㄱ }}{\stackrel{\text { J }}{*}}$ & $\stackrel{\vec{\lambda}}{\mathrm{i}}$ \\
\hline SUB & 离| & $\begin{array}{l}m \\
\infty \\
\stackrel{\forall}{r} \\
\end{array}$ & $\begin{array}{l}\text { ने } \\
\text { 걱 }\end{array}$ & $\begin{array}{l}\stackrel{\circ}{\circ} \\
\stackrel{\sim}{\sim}\end{array}$ & 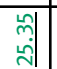 & $\begin{array}{l}\infty \\
\stackrel{\sim}{\rightarrow} \\
\infty \\
\sim\end{array}$ & 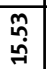 & $\underset{H}{\mathbb{N}}$ & 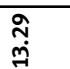 & 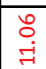 & $\begin{array}{l}\vec{m} \\
\vec{j}\end{array}$ & $\stackrel{n}{n}$ \\
\hline IND & $\underset{\sim}{\stackrel{\sim}{*}}$ & 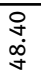 & $\begin{array}{l}m \\
\infty \\
\dot{\forall} \\
\dot{y}\end{array}$ & $\begin{array}{l}\hat{N} \\
\dot{m} \\
\dot{m}\end{array} \mid$ & \begin{tabular}{l|}
$\vec{b}$ \\
$\dot{m}$ \\
$\dot{m}$
\end{tabular} & $\begin{array}{l}\infty \\
0 \\
0 \\
\dot{q} \\
\dot{f}\end{array}$ & $\begin{array}{l}\vec{m} \\
\stackrel{\sim}{n}\end{array}$ & $\begin{array}{l}\$ \\
\infty \\
\infty\end{array}$ & 兴 & $\begin{array}{l}\hat{\hat{n}} \\
\text { aे }\end{array}$ & $\begin{array}{c}\vec{m} \\
\text { gे }\end{array}$ & $\stackrel{2}{9}$ \\
\hline$\vec{x}$ & 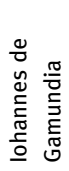 & 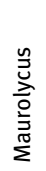 & $\stackrel{\frac{\pi}{0}}{=}$ & $\begin{array}{l}\stackrel{N}{\bar{E}} \\
\overline{\bar{G}}\end{array}$ & $\frac{\bar{\omega}}{\bar{\omega}}$ & $\begin{array}{l}\stackrel{u}{\tilde{n}} \\
\text { 苛 }\end{array}$ & 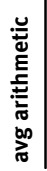 & $\frac{\vec{v}}{\tilde{w}}$ & 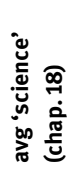 & 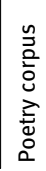 & 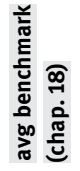 & $\frac{\overrightarrow{\vec{v}}}{\vec{n}}$ \\
\hline
\end{tabular}


Table 18: PoS values for the arithmetic corpus as percentages. Compare the values in table 12 from the general corpus.

\begin{tabular}{|c|c|c|c|c|c|c|c|}
\hline & $A D J$ & ADV & CONJ & $\mathrm{N}$ & PREP & PRON & V \\
\hline Martianus & $\underline{23.31}$ & 8.21 & 10.76 & $\underline{16.03}$ & 8.61 & 13.69 & $\underline{19.38}$ \\
\hline Boethius & $\underline{20.69}$ & 9.93 & 10.55 & $\underline{19.65}$ & 8.84 & $\underline{10.35}$ & 19.99 \\
\hline Isidore & $\underline{22.65}$ & 8.12 & 10.87 & $\underline{17.33}$ & 9.54 & 12.75 & $\underline{18.75}$ \\
\hline Ps-Bede & $\underline{14.53}$ & $\underline{7.25}$ & 9.07 & 22.78 & 9.76 & $\underline{10.05}$ & $\underline{26.57}$ \\
\hline Anxiomata & $\underline{21.43}$ & 7.93 & $\underline{12.22}$ & $\underline{17.29}$ & 9.31 & 12.61 & $\underline{19.21}$ \\
\hline Anonymus Toletanus, Regule & $\underline{18.82}$ & 8.32 & $\underline{11.28}$ & $\underline{18.24}$ & $\underline{10.66}$ & 11.07 & 21.62 \\
\hline Anonymus Siculus & $\underline{14.99}$ & $\underline{14.20}$ & 9.63 & $\underline{20.17}$ & $\underline{15.76}$ & $\underline{9.78}$ & $\underline{15.48}$ \\
\hline lordanes de Nemore & $\underline{19.78}$ & 7.97 & 12.87 & $\underline{10.34}$ & $\underline{17.85}$ & 10.96 & 20.22 \\
\hline Iohannes de Muris & $\underline{18.94}$ & 11.49 & 12.95 & $\underline{15.17}$ & 12.61 & 10.85 & $\underline{17.98}$ \\
\hline Albert of Saxony & $\underline{20.66}$ & 10.30 & 10.35 & $\underline{13.91}$ & $\underline{14.31}$ & 10.94 & $\underline{19.53}$ \\
\hline Oresmius & $\underline{20.70}$ & 8.24 & $\underline{12.86}$ & $\underline{17.10}$ & $\underline{11.68}$ & $\underline{10.63}$ & $\underline{18.79}$ \\
\hline Iohannes de Gamundia & $\underline{17.36}$ & $\underline{5.96}$ & 9.66 & 24.45 & $\underline{11.97}$ & $\underline{9.54}$ & 21.06 \\
\hline Maurolycus & $\underline{20.47}$ & 9.05 & $\underline{7.96}$ & $\underline{19.63}$ & $\underline{13.62}$ & $\underline{10.75}$ & $\underline{18.53}$ \\
\hline Vieta & $\underline{16.52}$ & $\underline{6.67}$ & 8.68 & 23.60 & $\underline{13.36}$ & $\underline{8.42}$ & 22.76 \\
\hline Leibniz & $\underline{16.48}$ & 8.41 & $\underline{14.16}$ & $\underline{16.42}$ & 9.42 & 11.00 & 24.10 \\
\hline Euler & 11.75 & 9.95 & 9.65 & $\underline{17.47}$ & $\underline{12.55}$ & 11.10 & $\underline{27.54}$ \\
\hline Gauss & $\underline{17.01}$ & 8.55 & 10.50 & $\underline{19.25}$ & $\underline{12.05}$ & 12.27 & 20.37 \\
\hline avg arithmetic & $\underline{18.59}$ & 8.86 & 10.82 & $\underline{18.17}$ & $\underline{11.88}$ & 10.99 & 20.70 \\
\hline stdev & 3.10 & 1.92 & 1.71 & 3.52 & 2.58 & 1.28 & 3.07 \\
\hline avg 'science' (chap. 18) & 12.36 & 9.74 & 9.86 & 24.49 & 9.13 & 12.55 & 21.87 \\
\hline Poetry corpus (chap. 18) & $\underline{15.52}$ & $\underline{7.23}$ & $\underline{7.28}$ & 30.54 & $\underline{4.38}$ & $\underline{10.49}$ & 24.57 \\
\hline avg benchmark (chap. 18) & 10.06 & 8.95 & 9.93 & 25.69 & 8.30 & 14.13 & 22.93 \\
\hline stdev & 0.87 & 0.53 & 0.43 & 1.40 & 0.77 & 1.12 & 1.03 \\
\hline
\end{tabular}

The same parameters and the same methodology were used as in the previous chapter, with the exception of the entropy measurement. As argued there, for texts shorter than 10,000 words such a measurement would not make sense, and many of the texts in this corpus are shorter. For the texts that are longer than 10,000 words, the entropy is $7.26 \pm 0.60$, thus significantly lower than in the other samples above. This indicates a linguistic monotony in these texts. 
\$4 The numbers show immediately that arithmetical Latin differs greatly from other prose, but within these texts less clear-cut subgroups are found. The benchmark texts are still expected to remain close together in PCA plots, and the metrical samples are expected to be furthest removed from this corpus's texts. Among the arithmetic texts, one might expect a grouping of pre-twelfth-century, scholastic-era, and Scientific-Revolution-era texts. First observations from the data in the tables show many characteristics that these texts together share with the general scientific sample, such as

- $\quad$ much higher (at least 3 stdevs) than usual: ADJ, ${ }^{17}$ PREP, NOM, ESSE, 3rd PAS, ADJ-SUF, modifiers;

- higher (at least 1 stdev) than usual: CONJ, ADJ-SUF;

- lower (at least 1 stdev) than usual: PRON, INF, GEN, ACC, ABL/DAT, 1st SG, ABL ABS;

- much lower than usual (at least 3 stdevs): N, PRON:POSS, entropy.

The following values contrast strongly with the general sample used in chapter 18: ADJ, PREP (more common); N, INF, entropy (lower). Among the arithmetic authors, post-Scientific-Revolution authors (Vieta, Leibniz, Euler, Gauß) use INF more normally, and early modern authors (Gamundia to Leibniz) use a lot of PTC.

The PCA graphic for the seven PoS (fig. 41) differentiates the arithmetic texts relatively well from the control groups and even more neatly from the poetry and Vulgate out-groups, the only exceptions being Ps-Bede and Euler. They also group quite neatly away from the average of thematic samples; the arithmetic average is closest to the medical texts. Unsurprisingly, the post-Scientific-Revolution authors tend to end up more in the centre of the plot, in the region of the 'new science' sample. The Euclid translation (Anonymus Siculus) is found at the lowerleft extreme, away from 'normal' Latin. Even a cursory glance at the tables above shows that the language of Ps-Bede differs markedly from the rest.

17 It should be noted that numerals are counted as ADJ. 


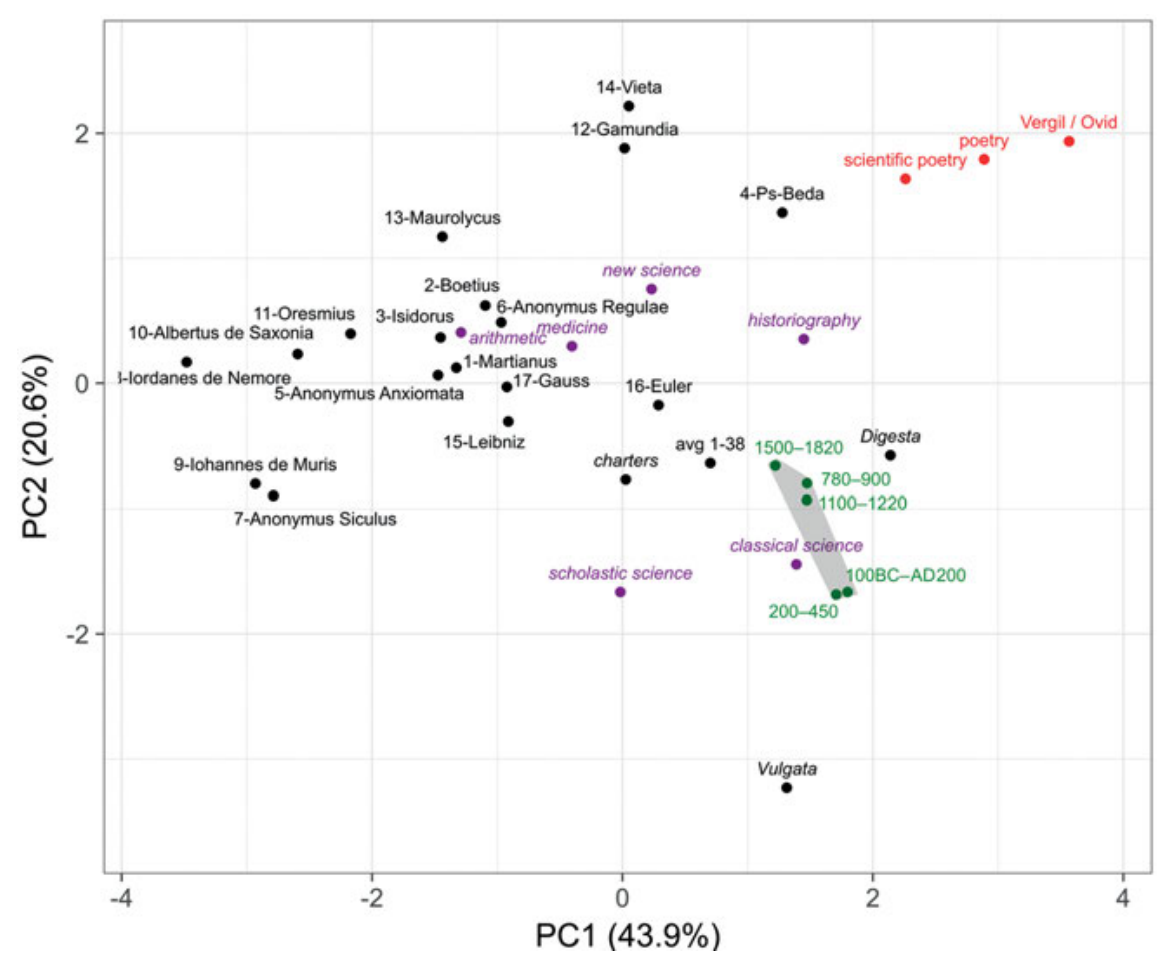

Fig. 41: PCA for the seven PoS.

If those parameters that differ more than 3 stdevs between the average of arithmetic and the benchmark are again chosen (ADJ, N, PREP, NOM, ESSE, PRON: POSS, 3rd PAS, ADJ-SUF, adding again 1st SG, and not using entropy for lack of reliable data), the result moves the arithmetic texts further away from the benchmark (fig. 42). Although the arithmetic texts still cluster neatly (again except PsBede), the scholastic sample now ends up among them. The early modern texts (11-15, except Euler and Gauß), again, have a tendency to form a subgroup among the arithmetic texts.

In all the plots, the general prose samples, the poetry samples, and the Vulgate are clearly differentiated from the arithmetic texts, with the exception of PsBede, a text that, indeed, differs quite markedly from the rest as it is much more of a merely didactic work than the others. For arithmetic texts, the PoS composition seems to differ more strongly from other kinds of Latin, including other scientific Latin. The Scientific Revolution authors Gamundia, Maurolycus, Vieta, and Leibniz (in one plot also Gauß) are usually close together. Euler seems to be an exception, using more conventional Latin. Somewhat surprisingly, in all cases pre- and post-twelfth-century authors could not be separated using these values. 


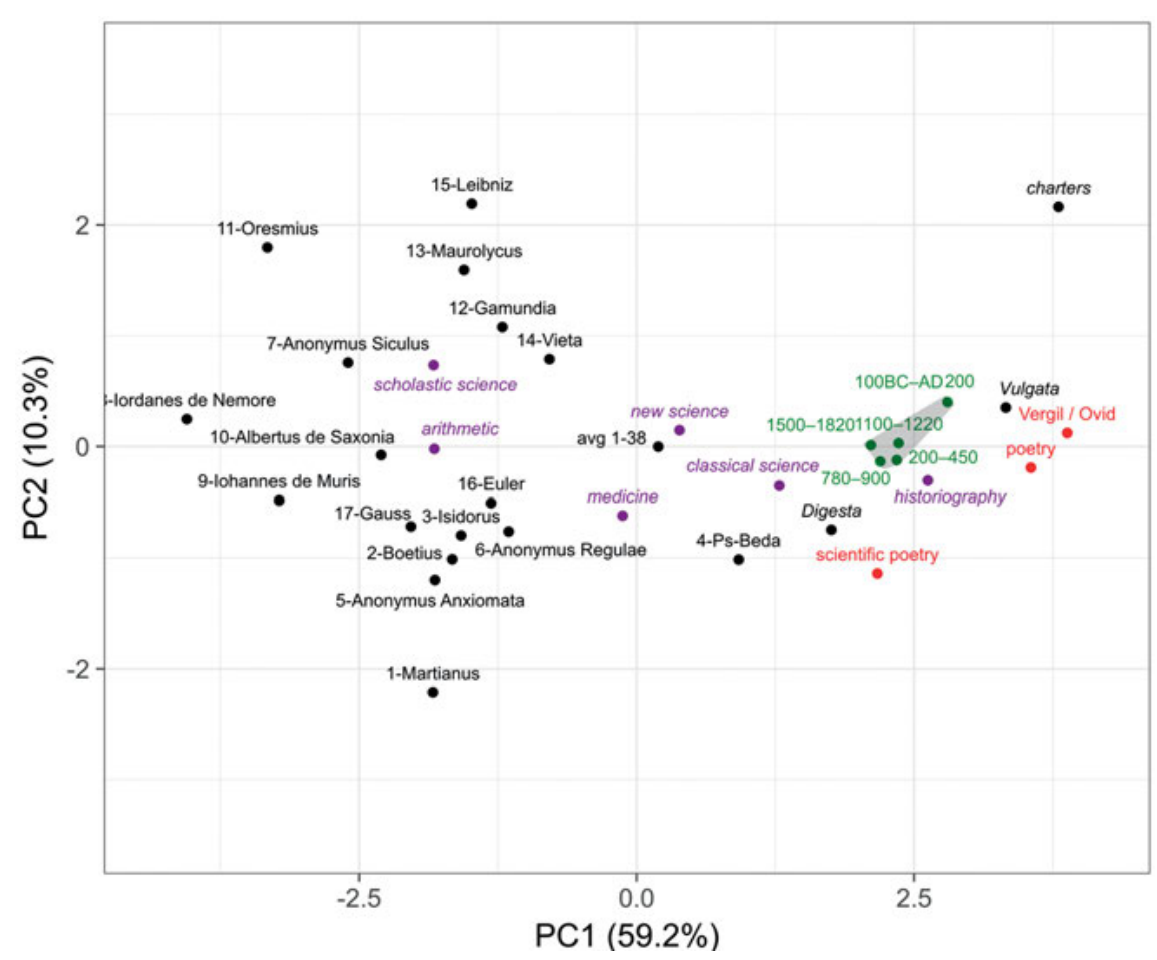

Fig. 42: PCA with the nine parameters with more than 3 stdevs average difference from benchmarks.

Finally, a stylometry plot based on the most common words using the same criteria as in the previous chapter is presented, this time only for words, not for lemmata. The tree in figure 43 corroborates the finding that the language of arithmetic differs strongly from that of the non-arithmetic texts used as out-groups. The groups within the arithmetic texts are much less pronounced, but it is conspicuous that the first Latin Euclid translation is very close to the root of all arithmetic texts. This shows nicely the enormous influence of Euclid's thought and diction on all later Latin writers. ${ }^{18}$ The pre-Euclidean texts (numbers 1-6) form a neat group, as do the mediaeval ones and the early modern ones (15-17).

18 It has been pointed out above that the texts older than Siculus are also heavily indebted to Euclid. 


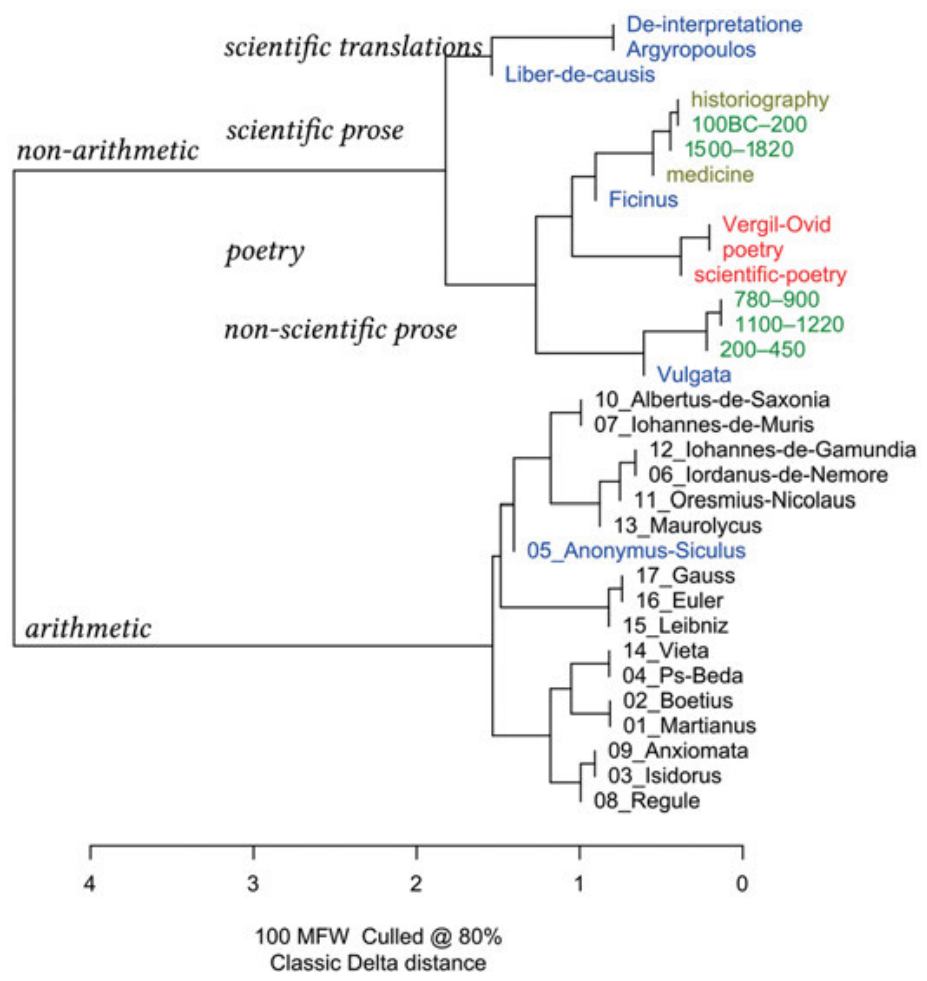

Fig. 43: Stylometry plot for the 100 most common words.

The results certainly do imply that arithmeticians - and possibly other 'hard' scientists within a clearly delimited field - used a rather uniform and unchanging type of language that contrasts strongly with other types of Latin. There are only few differences in the results based on the two different methods; this time, the stylometry plot seems to correspond even better to expectations. In contrast to the general sample in the previous chapter, there is a less strong caesura around the twelfth century - typical scholastics did not do arithmetic. But there is a strong dependence on Euclid's Elementa in general, which was translated fully into Latin by the anonymous Sicilian around 1175. Indeed, also in this field the amount and productivity of texts increases very significantly in the twelfth century.

\$5 Two more groups of less clearly scientific texts are now studied briefly, both of which would also seem to exhibit a rather uniform but different character linguistically: historiography and scientific/didactic hexametric poetry. For these, two corpora consisting of fifteen and ten texts respectively from republican times to modernity were defined. For the first, discursive prose texts about the history of 
a fixed topic were chosen; chronicles and similar more list-like texts were excluded. For both these groups of texts, it is less clear that they fulfil our criteria for science, as strictness of data and reasoning seems to be less crucial than rhetoric or poetic form for many writers. Above, the relation of historia to scientia was discussed (chap. 3 §5): it was stressed that some authors do use scientific approaches (e.g. Adam of Bremen in the sample), whereas others are rather propagandists (e.g. Liutprand). The data on the next pages show that these two groups of texts are quite homogeneous (although there are some atypical texts in the Middle Ages, especially Iohannes de Plano Carpini and Macer Floridus). The medical writers studied below (see chap. 21 and table 23) are also included in the data.

Table 19: The texts forming the two additional corpora on historiography and scientific poetry, a diachronic sample of fifteen and ten texts respectively.

\begin{tabular}{|c|c|c|c|c|}
\hline Author and work ${ }^{20}$ & Life dates & $\begin{array}{l}\text { Main scientific topic, in } \\
\text { modern terminology }\end{array}$ & $\begin{array}{l}\text { Number of } \\
\text { words, }{ }^{21} \\
\text { average word } \\
\text { length }\end{array}$ & $\begin{array}{l}\text { See } \\
\text { above }\end{array}$ \\
\hline $\begin{array}{l}\text { Sallust, Bellum lugurthinum, } \\
\text { ed. Ahlberg (1919) }\end{array}$ & $86-$ ca. 35 BC & $\begin{array}{l}\text { war against the Numidian } \\
\text { King Jugurtha }\end{array}$ & $\begin{array}{r}22 \\
6.04\end{array}$ & \multirow{8}{*}{$3 \S 5$} \\
\hline $\begin{array}{l}\text { Livy, } A b \text { urbe condita, } \\
\text { ed. Foster (1919) }\end{array}$ & ca. 59 BC-ca. AD 17 & history of the city of Rome & $\begin{array}{r}596 \\
6.69\end{array}$ & \\
\hline $\begin{array}{l}\text { Suetonius, De vita caesarum, } \\
\text { ed. Ihm (1907) }\end{array}$ & ca. $70-$ ca. 130 & $\begin{array}{l}\text { biography of Roman } \\
\text { emperors }\end{array}$ & $\begin{array}{r}72 \\
6.26\end{array}$ & \\
\hline $\begin{array}{l}\text { Rufinus, Historia monachorum } \\
\text { PL } 21 \text { (1879) }\end{array}$ & $345-411$ & history of monasticism & $\begin{array}{r}25 \\
5.77\end{array}$ & \\
\hline $\begin{array}{l}\text { Orosius, Historiae adversum } \\
\text { paganos, ed. Zangemeister } \\
(1882)\end{array}$ & $385-420$ & salvation history & $\begin{array}{r}78 \\
7.26\end{array}$ & \\
\hline $\begin{array}{l}\text { Gregory of Tours, Historiae, } \\
\text { ed. Krusch (1951) }\end{array}$ & $538-594$ & history of the Franks & $\begin{array}{r}123 \\
5.99\end{array}$ & \\
\hline $\begin{array}{l}\text { Notkerus Balbulus, Gesta Caroli } \\
\text { Magni, } \\
\text { ed. Haefele (1959) }\end{array}$ & ca. 840-912 & history of Charlemagne & $\begin{array}{r}17 \\
6.24\end{array}$ & \\
\hline $\begin{array}{l}\text { Liutprand of Cremona, } \\
\text { Antapodosis, } \\
\text { ed. Becker (1915) }\end{array}$ & $922-972$ & $\begin{array}{l}\text { polemical history of his } \\
\text { time }\end{array}$ & $\begin{array}{r}36 \\
6.12\end{array}$ & \\
\hline
\end{tabular}

19 Red means less than the average minus 1 stdev, green more than the average plus 1 stdev.

20 In thousands. The shortest texts are around 8,000 words; most are much longer. 
Table 19: (continued)

\begin{tabular}{|c|c|c|c|c|}
\hline Author and work ${ }^{20}$ & Life dates & $\begin{array}{l}\text { Main scientific topic, in } \\
\text { modern terminology }\end{array}$ & $\begin{array}{l}\text { Number of } \\
\text { words, }{ }^{21} \\
\text { average word } \\
\text { length }\end{array}$ & $\begin{array}{l}\text { See } \\
\text { above }\end{array}$ \\
\hline $\begin{array}{l}\text { Adam of Bremen, Gesta } \\
\text { Hammaburgensis ecclesiae } \\
\text { pontificum, } \\
\text { ed. Schmeidler (1917) }\end{array}$ & -1081 & $\begin{array}{l}\text { history of the Diocese of } \\
\text { Hamburg and the Far North }\end{array}$ & $\begin{array}{r}42 \\
6.08\end{array}$ & \multirow{7}{*}{$3 \S 5$} \\
\hline $\begin{array}{l}\text { William of Malmesbury, Gesta } \\
\text { regum Anglorum } \\
\text { PL } 179 \text { (1899) }\end{array}$ & ca. 1095 -ca. 1143 & Anglo-Saxon history & $\begin{array}{r}116 \\
6.22\end{array}$ & \\
\hline $\begin{array}{l}\text { lohannes de Plano Carpini, } \\
\text { Historia Mongalorum, } \\
\text { ed. Leonardi (1989) }\end{array}$ & ca. 1185-1252 & history of the Mongols & $\begin{array}{r}20 \\
5.50\end{array}$ & \\
\hline $\begin{array}{l}\text { Theodoricus de Niem, Historie de } \\
\text { gestis Romanorum principum, } \\
\text { ed. Colberg (1980) }\end{array}$ & $1340-1418$ & $\begin{array}{l}\text { history of the emperors of } \\
\text { the Holy Roman Empire }\end{array}$ & $\begin{array}{r}33 \\
6.09\end{array}$ & \\
\hline $\begin{array}{l}\text { Laurentius Valla, Gesta } \\
\text { Ferdinandi regis Aragonum, } \\
\text { ed. Besomi (1973) }\end{array}$ & $1406-1457$ & $\begin{array}{l}\text { history of Ferdinand I of } \\
\text { Aragon }\end{array}$ & $\begin{array}{r}42 \\
5.81\end{array}$ & \\
\hline $\begin{array}{l}\text { Nicola Orlandini, Historia } \\
\text { Societatis lesu } \\
\text { (Romae, 1615) }\end{array}$ & $1554-1606$ & history of the Jesuit Order & $\begin{array}{r}1,391 \\
6.53\end{array}$ & \\
\hline $\begin{array}{l}\text { lacobus Augustus Thuanus, } \\
\text { Historiae sui temporis } \\
\text { (Paris, 1606-1609) }\end{array}$ & $1553-1617$ & $\begin{array}{l}\text { history of the religious } \\
\text { wars from } 1545 \text { onward }\end{array}$ & $\begin{array}{r}1,279 \\
6.06^{21}\end{array}$ & \\
\hline
\end{tabular}

21 Average word length of these texts: $6.18 \pm 0.42$, somewhat longer than the benchmark: 5.95 \pm 0.32 . 
Table 19: (continued)

\begin{tabular}{|c|c|c|c|c|}
\hline Author and work ${ }^{20}$ & Life dates & $\begin{array}{l}\text { Main scientific topic, in } \\
\text { modern terminology }\end{array}$ & $\begin{array}{l}\text { Number of } \\
\text { words, } \\
\text { average word } \\
\text { length }\end{array}$ & $\begin{array}{l}\text { See } \\
\text { above }\end{array}$ \\
\hline $\begin{array}{l}\text { Lucretius, De rerum natura, } \\
\text { ed. Martin (1934) }\end{array}$ & ca. 99-ca. 55 BC & $\begin{array}{l}\text { natural philosophy, } \\
\text { Epicureanism }\end{array}$ & $\begin{array}{r}49 \\
5.58\end{array}$ & $8 \S 6$ \\
\hline $\begin{array}{l}\text { Manilius, Astronomica, } \\
\text { ed. van Wageningen (1916) }\end{array}$ & $\begin{array}{l}\text { early 1st century AD } \\
\text { (?) }\end{array}$ & astronomy and astrology & $\begin{array}{r}28 \\
5.77\end{array}$ & $5 \S 2$ \\
\hline $\begin{array}{l}\text { Anonymus, Aetna, } \\
\text { ed. Duff (1934) }\end{array}$ & before 79 & geology & $\begin{array}{r}4 \\
5.74\end{array}$ & $8 \S 8$ \\
\hline $\begin{array}{l}\text { Avienus Rufius Festus, Ora } \\
\text { maritima, } \\
\text { ed. Schulten (1922) }\end{array}$ & ca. $305-$ ca. 375 & geography & $\begin{array}{r}4 \\
5.80\end{array}$ & $5 \S 2$ \\
\hline $\begin{array}{l}\text { Walahfrid Strabo, Hortulus } \\
\text { PL } 114 \text { (1879) }\end{array}$ & ca. $808-849$ & botany & $\begin{array}{r}3 \\
5.95\end{array}$ & $5 \S 2$ \\
\hline $\begin{array}{l}\text { Macer Floridus, De viribus } \\
\text { herbarum, } \\
\text { ed. Baudet (1845) }\end{array}$ & 11th century & medical botany & $\begin{array}{r}14 \\
5.84\end{array}$ & $5 \S 2$ \\
\hline $\begin{array}{l}\text { Marcellus Palingenius Stellatus, } \\
\text { Zodiacus vitae, } \\
\text { ed. Chomarat (1996) }\end{array}$ & ca. $1500-1543$ & ethics, metaphysics, satire & $\begin{array}{r}66 \\
5.60\end{array}$ & $5 \S 2$ \\
\hline $\begin{array}{l}\text { Bruno, De monade, numero et } \\
\text { figura, } \\
\text { ed. Fiorentino (1879-1891) }\end{array}$ & $1548-1600$ & arithmology & $\begin{array}{r}10 \\
5.83\end{array}$ & $12 \S 3$ \\
\hline $\begin{array}{l}\text { Benedictus Stay, Philosophia } \\
\text { versibus tradita } \\
\text { (Romae, 1747) }\end{array}$ & $1714-1801$ & history of philosophy & $\begin{array}{r}82 \\
5.64\end{array}$ & $13 \S 4$ \\
\hline $\begin{array}{l}\text { Bernardus Zamagna, Navis aeria } \\
\text { (Romae, 1768) }\end{array}$ & $1735-1820$ & airships & $\begin{array}{r}13 \\
5.65^{22}\end{array}$ & $13 \S 4$ \\
\hline
\end{tabular}

22 Average word length of these texts: $5.91 \pm 0.49$, almost the same as the benchmark: $5.95 \pm 0.32$, but significantly longer than the poetry sample: 5.49 . 


\begin{tabular}{|c|c|c|c|c|c|c|c|c|c|c|c|c|c|}
\hline 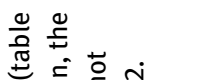 & Entropy & $\begin{array}{l}9 \\
a \\
a\end{array}$ & 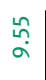 & ?ํํㅇ| & $\stackrel{\overbrace{}}{a}$ & $\begin{array}{l}\tilde{\sigma} \\
\alpha\end{array}$ & $\begin{array}{l}0 \\
⿱ \\
\infty \\
\infty\end{array} \mid$ & 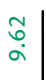 & $\begin{array}{l}\text { ț } \\
\text { aे }\end{array}$ & $\begin{array}{c}\stackrel{m}{\hat{n}} \\
\alpha\end{array}$ & 角 & 구 & 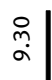 \\
\hline 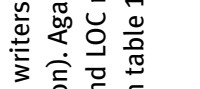 & ABL ABS & 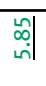 & $\vec{m} \mid$ & ㅊํ & $\begin{array}{l}\stackrel{0}{0} \\
\stackrel{N}{i}\end{array}$ & సี & 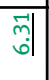 & $\underset{\substack{\mathcal{N} \\
m}}{\mathcal{N}}$ & 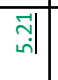 & $\begin{array}{l}\stackrel{m}{N} \\
\text { i }\end{array}$ & \begin{tabular}{l|l|} 
임 \\
$\infty$
\end{tabular} & $\vec{m}$ & 效| \\
\hline 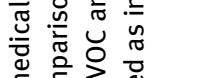 & Modifiers & @. & $\begin{array}{ll}0 \\
m \\
0\end{array}$ & वे. & 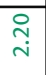 & $\begin{array}{ll}\infty \\
\infty \\
0 \\
0\end{array}$ & $\begin{array}{l}\text { oे } \\
\text { i }\end{array}$ & $\underset{\sim}{\stackrel{\sim}{*}}$ & 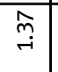 & $\underset{\sim}{\stackrel{N}{\rightarrow}}$ & $\begin{array}{l}m \\
\infty \\
\infty \\
0\end{array}$ & $\underset{\sim}{\stackrel{N}{i}}$ & 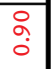 \\
\hline 눈 & N-SUF & $\stackrel{\hat{\alpha}}{\mathrm{i}}$ & $\begin{array}{l}\stackrel{n}{\hat{N}} \\
\stackrel{i}{i}\end{array}$ & $\begin{array}{c}\stackrel{N}{m} \\
m\end{array}$ & $\begin{array}{l}\infty \\
\stackrel{\infty}{m} \\
\dot{m}\end{array}$ & $\underset{\sim}{\tilde{N}}$ & (1) & \begin{tabular}{l|}
$\vec{f}$ \\
m. \\
\end{tabular} & 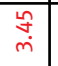 & 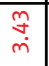 & 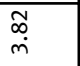 & $\stackrel{m}{m} \mid$ & શે \\
\hline 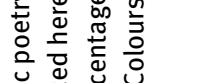 & ADJ-SUF & $\begin{array}{l}\hat{2} \\
\hat{\jmath} \\
0\end{array}$ & $\begin{array}{l}m \\
\stackrel{m}{m} \\
\dot{r}\end{array}$ & 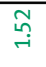 & $\begin{array}{l}m \\
\\
o \\
o\end{array}$ & $\underset{\sim}{\stackrel{5}{-}}$ & 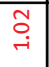 & 离 & 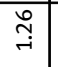 & $\begin{array}{ll}\vec{n} \\
\vec{r} \\
\vec{r}\end{array}$ & 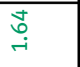 & ) & 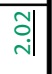 \\
\hline ڤ் & 3rd PAS & ঙ̧ & 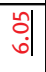 & 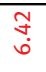 & 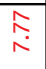 & $\stackrel{n}{\sim}$ & $\begin{array}{l}\vec{m} \\
\dot{0}\end{array}$ & \begin{tabular}{l|}
$\vec{y}$ \\
जीl
\end{tabular} & $\stackrel{\hat{N}}{\hat{\sigma}}$ & \begin{tabular}{l|l}
$m$ \\
0 \\
$\infty$ \\
$\infty$
\end{tabular} & चु & $\begin{array}{l}\text { @ } \\
\infty\end{array}$ & $\underset{\sim}{\infty}$ \\
\hline 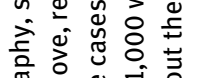 & 1st SG & $\begin{array}{l}\hat{n} \\
\dot{m}\end{array}$ & $\begin{array}{l}0 \\
\stackrel{+}{+} \\
i\end{array}$ & 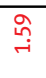 & $\begin{array}{l}\stackrel{8}{8} \\
i\end{array}$ & $\stackrel{\substack{m \\
i}}{ }$ & $\underset{P}{\vec{j}}$ & 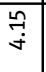 & 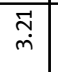 & $\begin{array}{ll}\infty \\
i \\
i\end{array}$ & $\stackrel{9}{9}$ & $\begin{array}{c}\text { mे. } \\
\text { o. }\end{array}$ & $\begin{array}{l}\vec{b} \\
\dot{\sim}\end{array}$ \\
\hline & \begin{tabular}{|l|} 
PRON:POSS \\
\end{tabular} & $\vec{m}$ & वे| & 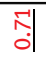 & $\underset{\rightarrow}{\stackrel{\sim}{\sim}}$ & श्रे & $\underset{\text { q }}{\mathrm{i}}$ & 齐 & $\stackrel{\infty}{+\infty}$ & 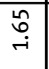 & $\underset{+}{\vec{j}}$ & $\begin{array}{l}0 \\
\infty \\
0 \\
0\end{array}$ & $\stackrel{\substack{\infty \\
\rightarrow \rightarrow}}{\rightarrow}$ \\
\hline 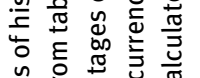 & \begin{tabular}{|l|} 
CONJ:S \\
\end{tabular} & $\begin{array}{l}\infty \\
\stackrel{\infty}{0} \\
i\end{array}$ & 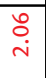 & 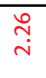 & \begin{tabular}{ll}
\multirow{2}{0}{} \\
$\stackrel{\sim}{*}$
\end{tabular} & 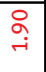 & 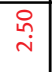 & 望 & $\stackrel{\circ}{\circ}$ & 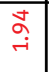 & $\overrightarrow{\vec{w}}$ & $\begin{array}{l}\infty \\
\stackrel{\infty}{\infty} \\
i\end{array}$ & 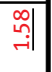 \\
\hline & REL & $\stackrel{\overrightarrow{7}}{m}$ & $\begin{array}{l}n \\
\stackrel{n}{i} \\
\end{array}$ & 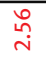 & $\stackrel{न}{\stackrel{f}{m}}$ & $\begin{array}{ll}0 \\
\dot{m} \\
i\end{array}$ & $\stackrel{\tilde{n}}{n}$ & 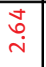 & $\begin{array}{l}a \\
\dot{m} \\
m\end{array}$ & $\begin{array}{ll}\& \\
\dot{f} \\
\end{array}$ & స్ল & $\begin{array}{l}\stackrel{\circ}{\circ} \\
\dot{m}\end{array}$ & $\stackrel{\substack{\infty \\
\sim}}{\sim}$ \\
\hline 焉 & \begin{tabular}{|l|} 
ESSE \\
\end{tabular} & $\begin{array}{l}\infty \\
\stackrel{\infty}{i} \\
i\end{array}$ & $\begin{array}{l}\text { N̦ } \\
\text { m }\end{array}$ & iㅛ & 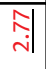 & ज़े & N્ત & \begin{tabular}{l|l|}
$m$ \\
$\infty$ \\
$\rightarrow \mid$
\end{tabular} & ㅇํํ| & 현 & 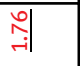 & 㟧 & ज्ञा \\
\hline$\stackrel{0}{I}$ & \begin{tabular}{|l}
$\mathrm{ABL} / \mathrm{DAT}$ \\
\end{tabular} & 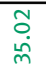 & $\begin{array}{l}\stackrel{\infty}{\infty} \\
\stackrel{m}{m} \\
\end{array}$ & 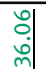 & $\begin{array}{l}\vec{i} \\
\vec{m}\end{array}$ & 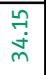 & $\begin{array}{l}0 \\
0 \\
\dot{m} \\
\text { m. }\end{array}$ & 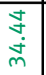 & $\begin{array}{l}\hat{a} \\
\hat{n} \\
m\end{array}$ & $\begin{array}{l}\stackrel{7}{7} \\
\dot{m} \\
\end{array}$ & $\begin{array}{l}\tilde{m} \\
\dot{m} \\
\dot{m}\end{array}$ & $\begin{array}{l}\stackrel{n}{\sim} \\
\stackrel{m}{m}\end{array}$ & \begin{tabular}{l|}
$\vec{j}$ \\
$\dot{m}$ \\
\end{tabular} \\
\hline 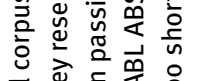 & ACC & 綮| & के & 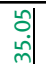 & $\begin{array}{l}\hat{n} \\
\vec{m} \\
\vec{m}\end{array}$ & $\begin{array}{l}\text { So } \\
\dot{2}\end{array}$ & $\begin{array}{l}0 \\
\dot{0} \\
\dot{m} \\
\text { n. }\end{array}$ & 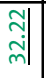 & $\begin{array}{l}\hat{a} \\
\infty \\
\infty\end{array}$ & 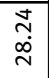 & 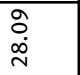 & श्र & $\begin{array}{l}\text { fे } \\
\text { จे }\end{array}$ \\
\hline 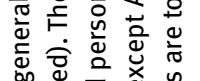 & GEN & $\begin{array}{l}\infty \\
\stackrel{\infty}{+} \\
\stackrel{q}{+}\end{array}$ & $\begin{array}{l}\infty \\
\stackrel{\infty}{n} \\
\stackrel{n}{n}\end{array}$ & $\begin{array}{l}\vec{j} \\
\dot{0} \\
\stackrel{1}{1}\end{array}$ & $\begin{array}{l}m \\
\stackrel{9}{a} \\
a\end{array}$ & $\begin{array}{l}\substack{n \\
\infty \\
\infty \\
\sim} \\
\end{array}$ & $\begin{array}{l}\vec{n} \\
\dot{H} \\
\end{array}$ & $\begin{array}{l}\text { Na } \\
\end{array}$ & $\begin{array}{l}\stackrel{ \pm}{N} \\
\stackrel{\sim}{\sim}\end{array}$ & $\begin{array}{l}\stackrel{2}{2} \\
\stackrel{2}{a}\end{array}$ & $\begin{array}{l}\text { qे } \\
\text { gे }\end{array}$ & 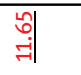 & 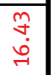 \\
\hline 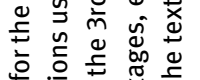 & NOM & $\begin{array}{l}\text { ำ } \\
\text { à }\end{array}$ & 월 & 离 & 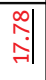 & \begin{tabular}{ll|} 
& 0 \\
0 \\
0 \\
\hdashline
\end{tabular} & ज़्वे| & $\begin{array}{l}\text { ปี } \\
\text { : }\end{array}$ & \begin{tabular}{|l|}
$\infty$ \\
$\infty$ \\
$\dot{\sim}$ \\
\end{tabular} & 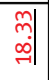 & $\begin{array}{l}\text { नी } \\
0 \\
0 \\
-1\end{array}$ & 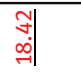 & $\begin{array}{l}\text { वे| } \\
\text { aे| }\end{array}$ \\
\hline 5 & \begin{tabular}{|l} 
PTC \\
\end{tabular} & 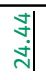 & 경 & 궜 & $\begin{array}{l}0 \\
\stackrel{0}{\omega} \\
\sim \\
\sim\end{array}$ & 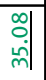 & 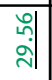 & \begin{tabular}{l|l} 
& $\infty$ \\
$\infty$ \\
$\dot{m}$
\end{tabular} & $\begin{array}{l}\overrightarrow{\vec{\sigma}} \\
\ddot{\sim}\end{array}$ & 究 & $\stackrel{m}{m} \mid$ & 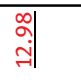 & 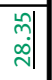 \\
\hline 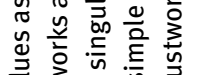 & INF & 学 & $\begin{array}{l}\stackrel{a}{+} \\
\dot{m} \\
\dot{q}\end{array}$ & $\begin{array}{l}\hat{o} \\
\dot{\theta} \\
\dot{i}\end{array}$ & $\begin{array}{l}\overrightarrow{\mathrm{J}} \\
\underset{\exists}{\mathrm{J}}\end{array}$ & 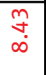 & $\begin{array}{ll} \\
\\
0 \\
\infty \\
\infty\end{array}$ & $\begin{array}{l} \\
\dot{y} \\
\dot{y}\end{array}$ & \begin{tabular}{|l|}
\multirow{i}{*}{} \\
\end{tabular} & $\begin{array}{l}\vec{\partial} \\
\dot{+} \\
\vec{i}\end{array}$ & ֶָ. & $\begin{array}{l}\vec{\infty} \\
0 \\
0 \\
\end{array}$ & \begin{tabular}{l|l|}
\multirow{2}{*}{} \\
$\infty$ \\
$\infty$
\end{tabular} \\
\hline 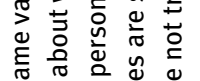 & SUB & 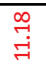 & 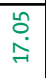 & $\begin{array}{l}\hat{N} \\
\hat{n}\end{array}$ & 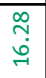 & $\begin{array}{l}\hat{0} \\
\dot{y}\end{array}$ & 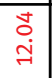 & 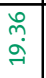 & \begin{tabular}{l|} 
\\
0 \\
$\dot{a}$ \\
$\dot{a}$
\end{tabular} & $\begin{array}{l}\overrightarrow{1} \\
0 \\
\vdots\end{array}$ & $\begin{array}{l}\text { Tु } \\
\stackrel{\sim}{\sim}\end{array}$ & $\begin{array}{l}\overrightarrow{+} \\
\vec{G}\end{array}$ & \begin{tabular}{l|}
$\stackrel{9}{+}$ \\
$\stackrel{+}{+}$
\end{tabular} \\
\hline 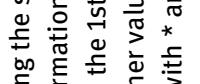 & IND & $\begin{array}{l}N \\
\tilde{m} \\
\vec{\forall}\end{array}$ & \begin{tabular}{l|} 
\\
0 \\
$m$ \\
$m$
\end{tabular} \mid & 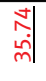 & 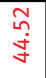 & $\begin{array}{l}\widehat{x} \\
\infty \\
\vec{\forall}\end{array}$ & $\begin{array}{l}\mathbb{N} \\
\hat{\sigma} \\
\stackrel{\sigma}{*}\end{array}$ & $\begin{array}{l}m \mid \\
\dot{\infty} \\
\dot{m}\end{array} \mid$ & \begin{tabular}{|l|} 
\\
0 \\
$\dot{\gamma}$ \\
$\dot{f}$
\end{tabular} & $\begin{array}{l}\stackrel{a}{+} \\
\stackrel{\infty}{+}\end{array}$ & $\begin{array}{l}\stackrel{9}{9} \\
\stackrel{9}{+}\end{array}$ & స̃| & \begin{tabular}{l}
\multirow{0}{*}{} \\
$\dot{+}$
\end{tabular} \\
\hline 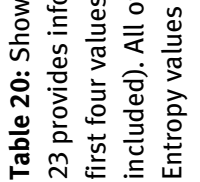 & 茊 & 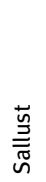 & $\stackrel{3}{3}$ & 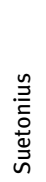 & 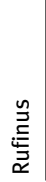 & $\begin{array}{l}\stackrel{n}{\bar{n}} \\
\stackrel{5}{0}\end{array}$ & 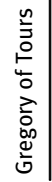 & $\begin{array}{l}\text { 产 } \\
\stackrel{ \pm}{0} \\
\text { L }\end{array}$ & 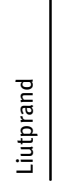 & 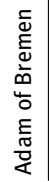 & 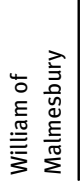 & 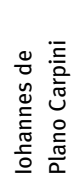 & 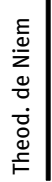 \\
\hline
\end{tabular}




\begin{tabular}{|c|c|c|c|c|c|c|c|c|c|c|c|c|c|c|c|c|}
\hline Entropy & $\begin{array}{l}\sigma \\
\sigma \\
\sigma\end{array}$ & 웡 & ๙ู & ₹ै & 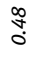 & $\begin{array}{l}0 \\
0 \\
\infty\end{array}$ & 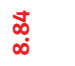 & $\stackrel{2}{2}$ & $\begin{array}{l}\stackrel{\circ}{0} \\
a\end{array}$ & $\begin{array}{l}\hat{a} \\
\hat{a}\end{array}$ & * & * & * & $\begin{array}{c}n \\
\swarrow \\
\infty\end{array}$ & 章 & $\begin{array}{l}\hat{0} \\
\stackrel{0}{ }\end{array}$ \\
\hline$A B L A B S$ & 윗 & \begin{tabular}{l}
\multirow{2}{*}{$\mid$} \\
|
\end{tabular} & \begin{tabular}{l|}
$a$ \\
o \\
-1
\end{tabular} & 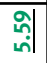 & 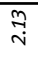 & \begin{tabular}{l}
\multirow{2}{*}{} \\
$\stackrel{+}{+}$
\end{tabular} & 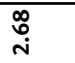 & $\stackrel{m}{\stackrel{m}{m}}$ & \begin{tabular}{l|l|}
$\overrightarrow{0}$ \\
$\dot{y}$
\end{tabular} & | & 司 & \begin{tabular}{l|l|}
0 \\
0 \\
0 \\
0
\end{tabular} & 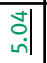 & $\begin{array}{l}\stackrel{\circ}{\circ} \\
\text { m. }\end{array}$ & $\begin{array}{l}\stackrel{m}{\stackrel{m}{m}} \\
\dot{m}\end{array}$ & $\begin{array}{l}\overrightarrow{\vec{b}} \\
\dot{m}\end{array}$ \\
\hline Modifiers & $\underset{\rightarrow}{\stackrel{J}{\rightarrow}}$ & 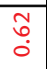 & กี้ & $\underset{7}{\stackrel{7}{7}}$ & 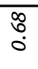 & ㅇํㅇ| & 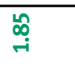 & $\stackrel{+}{+}$ & $\stackrel{\infty}{\stackrel{\infty}{\rightarrow}}$ & 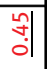 & \begin{tabular}{l|l} 
에 \\
:
\end{tabular} & 국 & 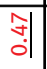 & 곙 & $\underset{\sim}{\stackrel{\sim}{\sim}}$ & $\begin{array}{l}\tilde{U} \\
\stackrel{0}{0}\end{array}$ \\
\hline N-SUF & $\begin{array}{l} \\
\infty \\
\infty \\
\sim\end{array}$ & 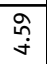 & $\underset{m}{\vec{f}}$ & $\stackrel{\sim}{\stackrel{n}{m}}$ & ôn & $\begin{array}{l}\stackrel{N}{\infty} \\
\dot{m}\end{array}$ & 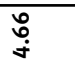 & $\underset{\mathrm{j}}{\mathrm{j}}$ & $\begin{array}{l}\overrightarrow{0} \\
0 \\
0\end{array}$ & 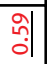 & $\begin{array}{l}0 \\
\vdots\end{array}$ & $\begin{array}{l}\text { त्ञ } \\
\text { In }\end{array}$ & \begin{tabular}{l|l|}
$\infty$ \\
$\vdots$ \\
\hdashline
\end{tabular} & \begin{tabular}{ll|} 
& 0 \\
0 & 0 \\
0
\end{tabular} & |윙 & $\begin{array}{l}\text { कीे } \\
\text { ते| }\end{array}$ \\
\hline ADJ-SUF & 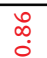 & $\begin{array}{l}\stackrel{N}{\sim} \\
\stackrel{H}{*}\end{array}$ & $\stackrel{\sim}{\sim}$ & $\underset{\sim}{\stackrel{n}{\sim}}$ & वे. & 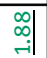 & 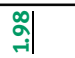 & $\stackrel{\sim}{\sim}$ & $\begin{array}{l}n \\
\stackrel{n}{o}\end{array}$ & $\stackrel{\text { f }}{\rightarrow}$ & 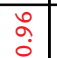 & \begin{tabular}{l|}
\multirow{i}{*}{} \\
J
\end{tabular} & \begin{tabular}{l|l|}
$\infty$ \\
$\stackrel{-}{|c|}$
\end{tabular} & 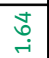 & $\underset{\text { స్ }}{\text { i }}$ & $\begin{array}{l}\vec{\sigma} \\
\stackrel{-}{r}\end{array}$ \\
\hline 3rd PAS & $\begin{array}{l}\overrightarrow{+} \\
\infty\end{array}$ & $\begin{array}{l}\vec{\infty} \\
\stackrel{\sim}{\sim}\end{array}$ & \begin{tabular}{l} 
\\
\hdashline \\
$\infty$ \\
$\infty$
\end{tabular} & 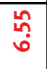 & 吕 & 귀ำ & $\begin{array}{l}\mathbf{n} \\
\stackrel{N}{\mathrm{j}}\end{array}$ & ఫ্ّ & $\begin{array}{l}\hat{n} \\
\stackrel{n}{\wedge} \\
\end{array}$ & 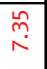 & ज्ञ & $\begin{array}{ll}\stackrel{n}{\sim} \\
\end{array}$ & $\begin{array}{l}\tilde{n} \\
\stackrel{n}{a} \\
\end{array}$ & 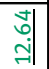 & $\begin{array}{l}\stackrel{m}{\infty} \\
\stackrel{\infty}{\wedge}\end{array}$ & \begin{tabular}{l} 
\\
\multirow{\circ}{*}{}
\end{tabular} \\
\hline 1st SG & $\stackrel{\vec{f}}{m}$ & $\begin{array}{l}\infty \\
\stackrel{\infty}{\rightarrow} \\
\rightarrow\end{array}$ & $\begin{array}{l}\vec{\sigma} \\
0\end{array}$ & $\vec{a}$ & ָ̊ & $\begin{array}{l}\text { m) } \\
\text { aोl }\end{array}$ & $\underset{\sim}{\stackrel{+}{*}}$ & $\underset{j}{J}$ & $\underset{⿱}{\stackrel{\sim}{i}}$ & \begin{tabular}{l}
9 \\
\multirow{2}{0}{} \\
\end{tabular} & $\begin{array}{l}n \\
\vdots \\
o\end{array}$ & \begin{tabular}{l|}
\multirow{2}{*}{} \\
$\stackrel{\text { in }}{ }$
\end{tabular} & 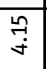 & 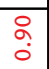 & 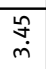 & $\begin{array}{l}\stackrel{\infty}{r}_{\rightarrow} \\
\end{array}$ \\
\hline PRON:POSS & $\begin{array}{l}\stackrel{0}{\sim} \\
\rightarrow \\
\rightarrow\end{array}$ & $\begin{array}{l}8 \\
\\
\end{array}$ & $\begin{array}{c}10 \\
0 \\
0 \\
\vdots\end{array}$ & $\begin{array}{l}\stackrel{\infty}{\rightarrow} \\
\rightarrow-1\end{array}$ & 皃 & $\begin{array}{l}\vec{i} \\
-i\end{array}$ & 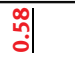 & นึ & 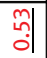 & \begin{tabular}{ll|l} 
& 0 \\
0 \\
0 \\
0
\end{tabular} & \begin{tabular}{l|l} 
\\
$\vdots$ \\
0
\end{tabular} & 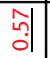 & \begin{tabular}{l|l|}
\multirow{2}{*}{} & \\
$\vdots$ &
\end{tabular} & 잉 & $\begin{array}{l}\text { Na. } \\
\vdots\end{array}$ & \begin{tabular}{l}
\multirow{2}{*}{} \\
$\vdots$
\end{tabular} \\
\hline CONJ:S & ने & $\begin{array}{l}\text { ?f } \\
\rightarrow\end{array}$ & ฏे। & $\underset{\text { i⿱ }}{\text { i }}$ & $\begin{array}{c}\tilde{m} \\
0 \\
0\end{array}$ & $\underset{\sim}{\stackrel{N}{\sim}}$ & ষ্ণ & న్ & $\stackrel{9}{\stackrel{9}{m}}$ & 亲 & 常 & 殸 & 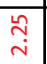 & $\overrightarrow{⿱ \vec{m}}$ & $\overrightarrow{\vec{N}}$ & $\begin{array}{l}\infty \\
\stackrel{\infty}{\sim} \\
\end{array}$ \\
\hline REL & $\vec{m}$ & $\begin{array}{l}\hat{O} \\
\dot{m}\end{array}$ & 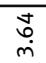 & 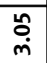 & वे & $\begin{array}{l}\underset{\sim}{\infty} \\
\stackrel{+}{\sim}\end{array}$ & İ & $\stackrel{\tilde{n}}{\tilde{m}}$ & $\underset{\substack{\infty \\
m}}{\infty}$ & $\stackrel{\substack{m\\
}}{ }$ & 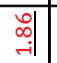 & $\begin{array}{l}\text { 吕 } \\
\text { नी }\end{array}$ & \begin{tabular}{l|}
$\infty$ \\
$\stackrel{\sim}{N}$ \\
\end{tabular} & 齐 & 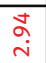 & $\begin{array}{l}\infty \\
\stackrel{\infty}{\sim} \\
\stackrel{i}{*}\end{array}$ \\
\hline ESSE & $\begin{array}{l}\stackrel{\tilde{n}}{\hat{n}} \\
m\end{array}$ & |त्ञ & 先| & \begin{tabular}{l}
\multirow{2}{*}{$\mid$} \\
|
\end{tabular} & $\hat{n}$ & 常 & 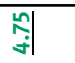 & $\underset{\dot{m}}{N}$ & 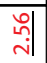 & वे| & i্ & 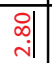 & \begin{tabular}{l|l|l|} 
& \\
o.
\end{tabular} & 웡 & 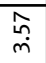 & 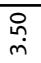 \\
\hline$\overline{A B L / D A T}$ & $\begin{array}{l}\vec{a} \\
\dot{m} \mid\end{array}$ & \begin{tabular}{l} 
\\
\multirow{J}{*}{} \\
$\stackrel{j}{*}$
\end{tabular} & $\begin{array}{l}\text { शे } \\
\text { aे }\end{array}$ & $\begin{array}{l}\tilde{N} \\
\stackrel{m}{m} \\
m\end{array}$ & $\underset{\sim}{\stackrel{N}{N}}$ & 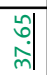 & 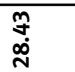 & 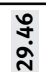 & $\begin{array}{l}\stackrel{9}{\rightarrow} \\
\vec{m}\end{array}$ & $\begin{array}{l}\infty \\
\stackrel{\infty}{h} \\
\infty \\
\sim \\
\sim\end{array}$ & $\begin{array}{l}\text { นิ } \\
\text { مे }\end{array}$ & $\begin{array}{l}\underset{\sim}{N} \\
\underset{\sim}{n}\end{array}$ & $\begin{array}{l}\text { D } \\
\stackrel{i}{i} \\
\tilde{m}\end{array}$ & \begin{tabular}{|l|}
$\infty$ \\
$\stackrel{\infty}{m}$ \\
$m$
\end{tabular} & $\begin{array}{l}\vec{N} \\
\stackrel{\infty}{\sim}\end{array}$ & $\begin{array}{l}m \\
\infty \\
\infty \\
\sim \\
\sim\end{array}$ \\
\hline $\mathrm{ACC}$ & 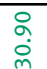 & $\begin{array}{l}\stackrel{0}{0} \\
\stackrel{0}{\infty} \\
\infty\end{array}$ & 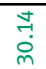 & $\begin{array}{l}\text { 苚 } \\
\text { ले }\end{array}$ & $\underset{-1}{\text { gे }}$ & 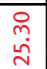 & $\begin{array}{l}\text { \& } \\
\text { ம் }\end{array}$ & 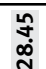 & $\begin{array}{l}\infty \\
0 \\
0 \\
0 \\
0\end{array}$ & 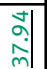 & $\begin{array}{l}\text { व } \\
\text { जे } \\
\text { m. }\end{array}$ & \begin{tabular}{l|l} 
of \\
p.
\end{tabular} & $\begin{array}{l}\underset{\sim}{*} \\
\underset{\sim}{\infty} \\
\sim\end{array}$ & \begin{tabular}{|l|}
$\hat{0}$ \\
$\dot{m}$ \\
$\dot{m}$
\end{tabular} & $\begin{array}{l}\stackrel{2}{\sim} \\
\stackrel{\sim}{N}\end{array}$ & 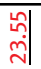 \\
\hline GEN & $\begin{array}{l}\stackrel{\sim}{\sim} \\
\ddot{n} \\
\end{array}$ & $\begin{array}{l}7 \\
\text { 국 }\end{array}$ & $\begin{array}{l}\text { 우 } \\
\stackrel{n}{n}\end{array}$ & 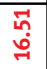 & $\underset{\sim}{\alpha}$ & 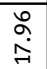 & $\begin{array}{l}\tilde{\infty} \\
\stackrel{\sim}{A}\end{array}$ & $\begin{array}{c}\infty \\
\underset{\infty}{\infty} \\
\infty\end{array}$ & $\begin{array}{l}\stackrel{+}{+} \\
\stackrel{\leftrightarrow}{\sim}\end{array}$ & 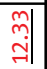 & $\begin{array}{l} \\
m \\
:\end{array}$ & 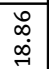 & 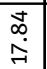 & \begin{tabular}{l|} 
ज्ञ \\
जे
\end{tabular} & 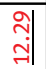 & $\begin{array}{l}\text { 도 } \\
\text { فी }\end{array}$ \\
\hline NOM & 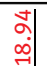 & 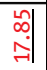 & $\begin{array}{l}\text { 命 } \\
\text { ปnd }\end{array}$ & \begin{tabular}{ll|}
$\infty$ \\
0 \\
0 \\
\hdashline
\end{tabular} & $\underset{\sim}{+}$ & 웍 & 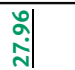 & $\underset{\sim}{\mathbb{N}}$ & 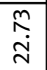 & \begin{tabular}{l}
\multirow{H}{*}{} \\
$\vec{A}$
\end{tabular} & 矛 & $\begin{array}{l}+ \\
\infty \\
\ddot{n} \\
\end{array}$ & \begin{tabular}{l}
\multirow{2}{*}{} \\
$\stackrel{-}{N}$
\end{tabular} & \begin{tabular}{|c}
$\hat{m}$ \\
$\stackrel{n}{v}$
\end{tabular} & $\begin{array}{c}\stackrel{\nabla}{\sim} \\
-\end{array}$ & $\begin{array}{l}\vec{a} \\
\vec{m}\end{array}$ \\
\hline $\begin{array}{l}\text { PTC } \\
\text {. }\end{array}$ & $\begin{array}{l}\vec{\sim} \\
\ddot{\sim} \\
\vec{\sim}\end{array}$ & 엥 & $\begin{array}{c}\hat{N} \\
\mathfrak{m}\end{array}$ & 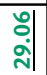 & \begin{tabular}{l}
\multirow{\forall}{*}{} \\
$\dot{\forall}$
\end{tabular} & $\begin{array}{l}\text { aे } \\
\dot{\sim}\end{array}$ & $\underset{\substack{F \\
\sim}}{\vec{f}}$ & 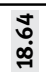 & 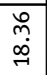 & \begin{tabular}{ll|} 
& \\
0 \\
0 \\
0
\end{tabular} & $\begin{array}{l}\text { aे } \\
\ddot{n}\end{array}$ & \begin{tabular}{l|} 
\\
$\infty$ \\
$\stackrel{\sim}{0}$ \\
\end{tabular} & \begin{tabular}{l|}
\multirow{N}{*}{} \\
d. \\
\end{tabular} & 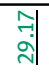 & $\begin{array}{l}\infty \\
\infty \\
\stackrel{+}{\sim} \\
\dot{\sim}\end{array}$ & $\begin{array}{l}\text { Na } \\
\stackrel{N}{N}\end{array}$ \\
\hline INF & $\begin{array}{l}\stackrel{O}{+} \\
\stackrel{+}{J}\end{array}$ & 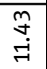 & $\begin{array}{l}\text { ñ } \\
\vdots \\
\vdots\end{array}$ & $\begin{array}{l}\stackrel{\text { qu}}{\mathrm{g}} \\
\text { (n) }\end{array}$ & 孛 & $\begin{array}{l} \\
\stackrel{8}{\circ} \\
\vdots \\
\end{array}$ & $\begin{array}{l}\stackrel{9}{+} \\
\text { ㄱ }\end{array}$ & $\underset{\text { \ี }}{\stackrel{\text { \} }{ }}} &{\text { 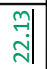 }} &{\begin{array}{l}\infty \\
\stackrel{\infty}{\infty} \\
\multirow{\infty}{*}{}\end{array}} &{\begin{array}{l}\text { స్ } \\
\text { I }\end{array}} &{\begin{array}{l}\underset{m}{\sim} \\
\stackrel{-}{-}\end{array}} &{\text { 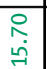 }} &{\begin{array}{l}\vec{\infty} \\
\vec{g}\end{array}} &{\begin{array}{l}\stackrel{u}{q} \\
\stackrel{\sim}{\sim}\end{array}} &{\text { 국 }} \\
{\hline \text { SUB }} &{\text { 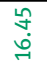 }} &{\text { 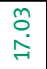 }} &{\text { 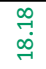 }} &{\begin{array}{l}\tilde{\infty} \\
\multirow{j}{*}{}\end{array}} &{\text { 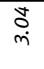 }} &{\begin{array}{l}N \\
\infty \\
0 \\
0\end{array}} &{\text { స్ }} &{\begin{array}{l}\vec{m} \\
\vec{f}\end{array}} &{\begin{array}{l}\mathbf{t} \\
\infty \\
\ddot{\eta}\end{array}} &{\begin{array}{|c|c|}\infty & \\
0 \\
\infty\end{array} \mid} &{\begin{array}{l}\text { nn } \\
m \\
\stackrel{n}{9}\end{array}} &{\text { 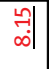 }} &{\text { 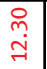 }} &{\begin{array}{c}\vdots \\
\vdots \\
\infty\end{array}} &{\begin{array}{l}\hat{m} \\
\vec{g}\end{array}} &{\begin{array}{l}\sqrt{1} \\
\hat{\sigma} \\
0\end{array}} \\
{\hline \text { IND }} &{\text { 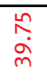 }} &{\text { 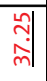 }} &{\begin{array}{l}\underbrace{\infty} \\
\vec{m}\end{array}} &{\text { 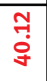 }} &{\begin{array}{l}8 \\
\dot{0} \\
\text { in }\end{array}} &{\text { 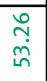 }} &{\text { 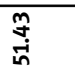 }} &{\begin{array}{l}\vec{m} \\
\stackrel{g}{g}\end{array}} &{\begin{array}{l} \\
\stackrel{\infty}{p} \\
\dot{\gamma}\end{array}} &{\begin{array}{l}\vec{i} \\
\vec{H}\end{array}} &{\begin{array}{l}\overrightarrow{\mathrm{U}} \\
\text { in }\end{array}} &{\begin{array}{l}\infty \\
0 \\
\infty \\
\infty \\
\infty\end{array}} &{\text { 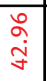 }} &{\begin{array}{|l|}\overrightarrow{9} \\
\stackrel{+}{~}\end{array}} &{\text { 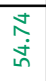 }} &{\begin{array}{l}\text { m̂n } \\
\hat{c} \\
\text { in }\end{array}} \\
{\hline \vec{x}} &{\frac{\sqrt[\pi]{0}}{\sqrt{\pi}}} &{\begin{array}{l}\overline{\bar{E}} \\
\overline{\overline{0}} \\
\overline{\frac{\pi}{2}} \\
\overline{0}\end{array}} &{\text { 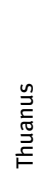 }} &{\underset{\pi}{\infty \infty}} &{\overrightarrow{\vec{v}}} &{\begin{array}{l}\frac{n}{0} \\
\frac{N}{\pi} \\
\frac{5}{U}\end{array}} &{\text { 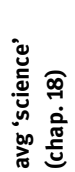 }} &{\text { 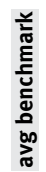 }} &{\text { 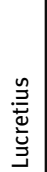 }} &{\text { 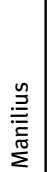 }} &{\text { 疍 }} &{\text { 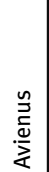 }} &{\begin{array}{l}\frac{\pi}{2} \\
\frac{\sqrt{n}}{\frac{\pi}{\pi}} \\
\frac{\pi}{3}\end{array}} &{\text { 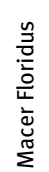 }} &{\text { 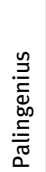 }} &{\text { 壳 }} \\
$\hline
\end{tabular}




\begin{tabular}{|c|c|c|c|c|c|c|c|c|c|c|c|c|c|c|c|c|c|}
\hline Entropy & ๙ุ. & $\begin{array}{l}\text { 경| } \\
\text { 에 }\end{array}$ & $\begin{array}{l}\infty \\
\stackrel{0}{\circ} \\
\stackrel{0}{ }\end{array}$ & 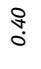 & ๙ิ| & 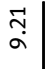 & * & $\begin{array}{c}\stackrel{2}{2} \\
\text { ○ी }\end{array}$ & ণ্ণু & $\begin{array}{l}\text { वे } \\
\text { aे }\end{array}$ & $\begin{array}{l}\text { fे } \\
\stackrel{a}{a}\end{array}$ & 움 & $\begin{array}{c}\tilde{\hat{n}} \\
0\end{array}$ & \begin{tabular}{l}
$\infty$ \\
$m$ \\
$\vdots$ \\
\hdashline
\end{tabular} \mid & $\begin{array}{l}0 \\
\infty \\
\infty \\
\infty\end{array}$ & $\stackrel{?}{a}$ & ণิ \\
\hline ABL ABS & \begin{tabular}{c}
$\infty$ \\
\hdashline \\
\hdashline
\end{tabular} \mid & 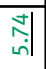 & 誉 & $\underset{\sim}{\mathbb{i}}$ & $\begin{array}{l}\text { 워 } \\
\text { ผी }\end{array}$ & \begin{tabular}{l}
$\infty$ \\
\multirow{y}{*}{$\mid$}
\end{tabular} & \begin{tabular}{l|} 
맘 \\
|
\end{tabular} & 익 & $\begin{array}{l}m \\
\hat{m} \\
\dot{m}\end{array}$ & $\begin{array}{l}\underset{\sim}{m} \\
\dot{m}\end{array}$ & 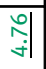 & $\begin{array}{l}\stackrel{\circ}{b} \\
\dot{m}\end{array}$ & $\underset{\sim}{n}$ & 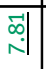 & 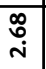 & $\stackrel{m}{\dot{m}}$ & $\begin{array}{l}\overrightarrow{7} \\
0\end{array}$ \\
\hline Modifiers & $\begin{array}{l}\vec{\alpha} \\
\vdots\end{array}$ & $\begin{array}{l} \\
\stackrel{0}{0} \\
\stackrel{0}{0}\end{array}$ & \begin{tabular}{l}
\multirow{2}{*}{$\hat{\circ}$} \\
$\stackrel{0}{\circ}$
\end{tabular} & $\begin{array}{c}\stackrel{m}{0} \\
\text { oे }\end{array}$ & 斊 & 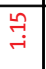 & 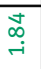 & 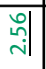 & $\underset{\sim}{\stackrel{ \pm}{\rightarrow}}$ & $\begin{array}{l}\stackrel{\circ}{\circ} \\
\stackrel{-}{+}\end{array}$ & $\begin{array}{l}\text { ำ } \\
\vdots\end{array}$ & $\begin{array}{l}\text { fo } \\
\stackrel{+}{*}\end{array}$ & $\begin{array}{l}\hat{N} \\
\text { O }\end{array}$ & \begin{tabular}{l|}
\multirow{1}{*}{} \\
$\stackrel{0}{0}$ \\
\end{tabular} & $\begin{array}{l}\infty \\
\infty \\
+i \\
+i\end{array}$ & $\begin{array}{l}0 \\
\stackrel{+}{*}\end{array}$ & \begin{tabular}{l} 
D \\
\multirow{0}{0}{}
\end{tabular} \\
\hline N-SUF & 齐 & \begin{tabular}{l|}
$\infty$ \\
$\stackrel{2}{0}$ \\
$\vdots$
\end{tabular} & \begin{tabular}{ll|} 
& 0 \\
0 \\
0 \\
0
\end{tabular} & $\sum_{0}^{\infty}$ & \begin{tabular}{l|l} 
\\
00 \\
0
\end{tabular} & गٕ: & $\begin{array}{l}\tilde{N} \\
\dot{m}\end{array}$ & 呫 & \begin{tabular}{l|l} 
\\
\multirow{1}{*}{$\mid$} \\
+1
\end{tabular} & $\begin{array}{l}\stackrel{D}{n} \\
\dot{m}\end{array}$ & $\begin{array}{l}\tilde{n} \\
\tilde{m}\end{array}$ & \begin{tabular}{l}
\multirow{\infty}{*}{} \\
$\stackrel{N}{N}$
\end{tabular} & $\widehat{\vec{r}}$ & 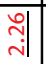 & $\begin{array}{l} \\
\stackrel{0}{x} \\
\dot{x}\end{array}$ & $\stackrel{?}{+}$ & $\begin{array}{l}\overline{2} \\
0\end{array}$ \\
\hline ADJ-SUF & $\begin{array}{l}\stackrel{N}{\stackrel{1}{0}} \\
\vdots\end{array}$ & ने & $\underset{\rightarrow}{\mathbb{T}}$ & $\begin{array}{c}\text { ले } \\
\text { हे }\end{array}$ & 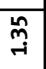 & 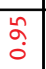 & $\begin{array}{l}\stackrel{N}{N} \\
\hat{0}\end{array}$ & 곙 & 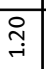 & $\begin{array}{l}\stackrel{n}{\sim} \\
\rightarrow \\
\rightarrow\end{array}$ & \begin{tabular}{l|} 
\\
\\
\hdashline \\
$\dot{f} \mid$
\end{tabular} & $\underset{\rightarrow}{\mathbb{A}}$ & $\underset{\sim}{\mathbb{N}}$ & @़|्|| & \begin{tabular}{l|}
$\infty$ \\
$\stackrel{\sim}{|c|} \mid$
\end{tabular} & $\underset{\sim}{\stackrel{i}{*}}$ & $\begin{array}{l}A \\
0\end{array}$ \\
\hline 3rd PAS & $\begin{array}{l}\stackrel{?}{r} \\
\stackrel{1}{r}\end{array}$ & $\begin{array}{l}\stackrel{m}{m} \\
\dot{f} \mid\end{array}$ & 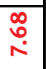 & $\underset{\sim}{\tilde{N}}$ & 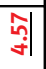 & $\begin{array}{l}m \\
\infty \\
0 \\
\dot{A}\end{array}$ & $\begin{array}{l}\alpha \\
\alpha \\
\infty\end{array}$ & 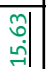 & \begin{tabular}{l|}
\multicolumn{2}{|c|}{} \\
$\infty$ \\
$\mid$
\end{tabular} & $\begin{array}{l}\text { mे } \\
\text { in }\end{array}$ & 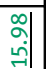 & $\begin{array}{l}\text { ît } \\
\text { ज्ञ }\end{array}$ & $\begin{array}{l}\vec{a} \\
\dot{m}\end{array}$ & \begin{tabular}{l|} 
\\
nొl \\
mll
\end{tabular} & \begin{tabular}{l|} 
กิ \\
\multirow{2}{*}{$\mid$}
\end{tabular} & ন্: & $\begin{array}{l}\text { वे } \\
\text { o }\end{array}$ \\
\hline 1st SG & $\stackrel{\stackrel{m}{\sim}}{i}$ & $\begin{array}{l}\tilde{N} \\
\stackrel{N}{N}\end{array}$ & 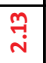 & $\stackrel{n}{\sim}$ & $\underset{\sim}{\stackrel{m}{n}}$ & 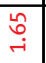 & $\begin{array}{l}\widehat{T} \\
\dot{\infty} \\
\dot{0}\end{array}$ & $\begin{array}{l}m \\
\\
\stackrel{i}{i}\end{array}$ & $\begin{array}{l}\vec{J} \\
\dot{m}\end{array}$ & $\begin{array}{l}\text { Oे } \\
\text { +i }\end{array}$ & $\begin{array}{l}\infty \\
\stackrel{\infty}{\sim} \\
\stackrel{\sim}{i}\end{array}$ & 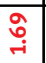 & $\widehat{\partial}$ & $\begin{array}{l}\underset{\sim}{N} \\
\rightarrow\end{array}$ & 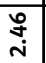 & \begin{tabular}{l}
\multirow{g}{*}{} \\
$\dot{y}$
\end{tabular} & $\stackrel{m}{n}$ \\
\hline PRON:POSS & $\begin{array}{l}1 \\
0 \\
0 \\
0\end{array}$ & $\begin{array}{l}0 \\
0 \\
0 \\
0\end{array}$ & 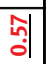 & 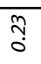 & 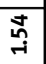 & 学 & 곙 & \begin{tabular}{l|l|} 
\\
ㄱ|
\end{tabular} & \begin{tabular}{ll|} 
& $m$ \\
0 \\
0
\end{tabular} \mid & 깅 & $\begin{array}{l} \\
\vdots\end{array}$ & \begin{tabular}{l|} 
\\
गे|
\end{tabular} & ?: & $\begin{array}{c}\vec{m} \\
\vdots \\
\vdots\end{array}$ & 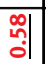 & مُ & กี \\
\hline CONJ:S & 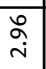 & $\begin{array}{l}\hat{0} \\
\dot{i}\end{array}$ & $\begin{array}{l}\text { N } \\
\text { N }\end{array}$ & $\begin{array}{l}\text { શิ } \\
0\end{array}$ & 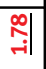 & $\begin{array}{l}\hat{n} \\
\dot{m}\end{array}$ & $\begin{array}{l}\circ \\
\infty \\
\dot{m}\end{array}$ & $\begin{array}{l}\text { गै। } \\
\dot{\forall} \mid\end{array}$ & $\begin{array}{l}\vec{J} \\
\stackrel{\rightarrow}{\rightarrow}\end{array}$ & $\begin{array}{l}\infty \\
\stackrel{\infty}{ } \\
i\end{array}$ & $\begin{array}{c}m \\
0 \\
\rightarrow \mid\end{array}$ & \begin{tabular}{l}
\multirow{m}{*}{} \\
\end{tabular} & $\begin{array}{l} \\
\\
- \\
-1\end{array}$ & \begin{tabular}{l|} 
게 \\
|
\end{tabular} & $\begin{array}{l}\dot{m} \\
\end{array}$ & న్ & के \\
\hline REL & $\begin{array}{l} \\
\stackrel{P}{m}\end{array}$ & $\begin{array}{l}m \\
\infty \\
\rightarrow \mid \\
\rightarrow \mid\end{array}$ & ले & 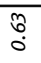 & 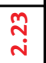 & $\underset{\dot{m}}{\stackrel{m}{m}}$ & ำำ & $\underset{\sim}{\sim}$ & 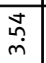 & $\underset{\sim}{\stackrel{\sim}{m}}$ & \begin{tabular}{l} 
\\
\\
\multirow{r}{*}{$\mid$}
\end{tabular} & $\begin{array}{l}\tilde{n} \\
\stackrel{N}{N}\end{array}$ & $\grave{o}$ & 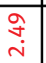 & $\begin{array}{l} \pm \\
\text { m. }\end{array}$ & $\begin{array}{c}m \\
\stackrel{m}{m}\end{array}$ & $\begin{array}{l} \\
\stackrel{2}{0} \\
0\end{array}$ \\
\hline ESSE & $\begin{array}{ll} & \\
\text { il }\end{array}$ & \begin{tabular}{|c|}
\multicolumn{1}{|c}{} \\
$\vdots$ \\
\end{tabular} & $\begin{array}{c}m \\
\text { N. }\end{array}$ & $\stackrel{m}{o}$ & व્సे & $\begin{array}{l}\text { जी } \\
\text { जी }\end{array}$ & $\begin{array}{c}\infty \\
\infty \\
\infty \\
\dot{m}\end{array}$ & 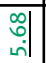 & \begin{tabular}{l|l|} 
& त्| \\
|
\end{tabular} & $\begin{array}{l}\stackrel{\infty}{\sim} \\
\stackrel{m}{m}\end{array}$ & $\stackrel{(7}{\sim} \mid$ & $\begin{array}{l}\hat{\circ} \\
\dot{m}\end{array}$ & $\begin{array}{l}\hat{n} \\
+i\end{array}$ & 곅| & 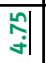 & $\underset{m}{N}$ & ণิ \\
\hline ABL/DAT & $\begin{array}{l}\stackrel{\circ}{i} \\
\stackrel{m}{m}\end{array}$ & 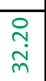 & $\begin{array}{l}n \\
\stackrel{n}{n} \\
\stackrel{m}{n}\end{array}$ & $\stackrel{\vec{P}}{m}$ & $\begin{array}{l}\infty \\
\stackrel{\infty}{0} \\
\stackrel{m}{m}\end{array}$ & $\begin{array}{l}\stackrel{n}{7} \\
\vec{m}\end{array}$ & $\begin{array}{l}\overrightarrow{0} \\
\dot{m}\end{array}$ & $\begin{array}{l}\text { No } \\
\dot{n} \\
\end{array}$ & 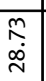 & 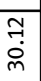 & 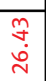 & $\begin{array}{l}\text { గ̂. } \\
\text { ని }\end{array}$ & $\underset{m}{\tilde{m}}$ & $\begin{array}{l}\text { ओ } \\
\infty \\
\text { m) }\end{array}$ & $\begin{array}{l}\stackrel{n}{f} \\
\stackrel{\infty}{\infty}\end{array}$ & 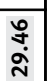 & $\underset{\sim}{\stackrel{P}{q}}$ \\
\hline $\mathrm{ACC}$ & $\begin{array}{l}\text { वे } \\
\text { aे }\end{array}$ & $\begin{array}{l}\stackrel{2}{2} \\
\stackrel{\sim}{*}\end{array}$ & $\begin{array}{l}\hat{n} \\
\hat{p}\end{array}$ & $\begin{array}{l}\vec{a} \\
\stackrel{m}{n}\end{array}$ & $\begin{array}{l}\text { å } \\
\text { స్ }\end{array}$ & 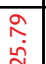 & $\underset{\substack{\infty \\
\infty \\
\infty}}{ }$ & 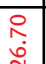 & $\begin{array}{l}\text { 영 } \\
\vdots\end{array}$ & $\begin{array}{l}\hat{\kappa} \\
b \\
b\end{array}$ & $\underset{\infty}{\stackrel{f}{\infty}}$ & \begin{tabular}{l}
2 \\
\multirow{6}{0}{}
\end{tabular} & $\underset{\sim}{\mathbb{T}}$ & 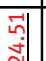 & 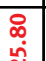 & $\begin{array}{l}\mathfrak{f} \\
\infty\end{array}$ & $\underset{+}{\stackrel{8}{0}}$ \\
\hline GEN & 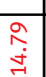 & $\begin{array}{l}\stackrel{ \pm}{\sim} \\
\ddot{g}\end{array}$ & $\begin{array}{l}\vec{F} \\
\text { J्न }\end{array}$ & นึ & 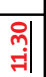 & $\begin{array}{l}\mathcal{I} \\
\text { \& } \\
\text { J }\end{array}$ & $\begin{array}{l}\hat{0} \\
\dot{\sim} \\
\vec{\sim}\end{array}$ & 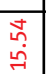 & \begin{tabular}{l|} 
\\
$\vdots$ \\
$\vdots$ \\
$\vdots$
\end{tabular} & 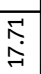 & $\begin{array}{l}\text { नी } \\
\stackrel{\sim}{\circ}\end{array}$ & 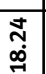 & হి & 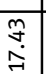 & 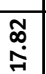 & $\underset{\substack{\infty \\
\infty}}{\infty}$ & $\begin{array}{l}\text { 吕 } \\
\stackrel{\sim}{n}\end{array}$ \\
\hline NOM & 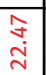 & $\begin{array}{l}\circ \\
\infty \\
\dot{\sim}\end{array}$ & $\begin{array}{l}\infty \\
0 \\
\dot{f} \\
\text { N }\end{array}$ & $\overline{\check{J}}$ & $\begin{array}{l}\vec{m} \\
\text { 㟧 }\end{array}$ & 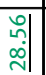 & $\begin{array}{l}0 \\
\text { oे } \\
\dot{\mathbf{N}}\end{array}$ & 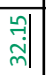 & \begin{tabular}{l|}
$\vec{b}$ \\
$\vdots$ \\
$\vdots$
\end{tabular} & 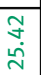 & $\begin{array}{l}8 \\
\text { ¿े } \\
\text { in }\end{array}$ & $\begin{array}{l}\text { 守 } \\
\text { 㟧 }\end{array}$ & \begin{tabular}{l}
\multirow{y}{*}{} \\
$\forall$
\end{tabular} & \begin{tabular}{l|} 
\\
aेd
\end{tabular} & \begin{tabular}{l|} 
\\
\multirow{2}{*}{} \\
ते
\end{tabular} & $\begin{array}{c}\vec{N} \\
\tilde{N} \\
\end{array}$ & : \\
\hline PTC & $\begin{array}{l}\vec{y} \\
\dot{+} \\
\vec{N}\end{array}$ & $\begin{array}{l}\text { ने } \\
\text { ○े }\end{array}$ & 웜 & $\begin{array}{l}\infty \\
\text { in } \\
\text { in }\end{array}$ & $\begin{array}{l} \\
\check{\nu} \\
\end{array}$ & $\begin{array}{l} \\
\dot{0} \\
\dot{b} \\
\end{array}$ & 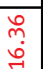 & $\begin{array}{l} \\
\stackrel{\infty}{\sim} \\
\stackrel{\sim}{\sim}\end{array}$ & \begin{tabular}{l|}
$m$ \\
0 \\
\hdashline
\end{tabular} & 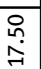 & $\begin{array}{l} \\
\stackrel{\sim}{~} \\
\text { d }\end{array}$ & $\begin{array}{l}m \\
\tilde{m} \\
\stackrel{N}{े}\end{array}$ & $\begin{array}{l} \\
\infty \\
0 \\
0\end{array}$ & 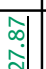 & $\begin{array}{c}\overrightarrow{5} \\
\substack{\infty \\
-1}\end{array}$ & 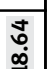 & 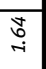 \\
\hline INF & 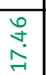 & 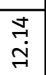 & 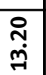 & $\underset{\forall}{7}$ & $\begin{array}{l}\infty \\
\stackrel{\infty}{0} \\
\stackrel{\sim}{0}\end{array}$ & 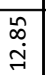 & $\begin{array}{l}\vec{I} \\
\vec{g}\end{array}$ & $\begin{array}{l}\stackrel{2}{a} \\
\infty\end{array}$ & $\begin{array}{l}\vec{J} \\
\sigma^{\circ}\end{array}$ & 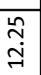 & 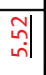 & $\begin{array}{l}\infty \\
\infty \\
\alpha \\
\alpha\end{array}$ & 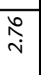 & $\begin{array}{l}\hat{\widehat{A}} \\
\dot{G}\end{array}$ & 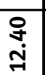 & 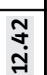 & aे \\
\hline SUB & $\begin{array}{l}\vec{m} \\
\grave{g}^{-}\end{array}$ & $\begin{array}{l}\hat{n} \\
\hat{n} \\
\hat{n}\end{array}$ & 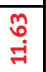 & 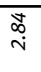 & $\begin{array}{l}\stackrel{2}{ } \\
\text { ت̇ }\end{array}$ & $\begin{array}{l}\text { กิ } \\
\text { مू }\end{array}$ & $\begin{array}{l}\text { 志 } \\
\text { مैं }\end{array}$ & $\begin{array}{l} \\
\dot{y} \\
\dot{y}\end{array}$ & \begin{tabular}{l|}
$\overrightarrow{\tilde{n}}$ \\
$m$ \\
$m$
\end{tabular} & $\begin{array}{l}\hat{m} \\
\dot{b}^{\circ}\end{array}$ & $\begin{array}{l}0 \\
\stackrel{\infty}{0} \\
\stackrel{-}{0}\end{array}$ & $\begin{array}{l}\vec{N} \\
\ddot{n}\end{array}$ & hूa & \begin{tabular}{l|}
\multirow{2}{*}{} \\
$\stackrel{\sigma}{\circ}$
\end{tabular} & $\begin{array}{l}\text { त्रे } \\
\text { m}\end{array}$ & $\begin{array}{l}\vec{m} \\
\dot{d}\end{array}$ & $\underset{\sim}{n}$ \\
\hline IND & $\begin{array}{l} \\
\stackrel{0}{+} \\
\dot{q}\end{array}$ & $\begin{array}{l}\text { ఏे } \\
\grave{j}\end{array}$ & \begin{tabular}{l}
\multirow{2}{\alpha}{} \\
$\tilde{\sigma}$
\end{tabular} & $\begin{array}{l} \\
0 \\
0\end{array}$ & $\begin{array}{l}\hat{\sim} \\
\text { oे }\end{array}$ & 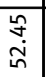 & 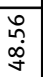 & 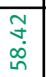 & $\begin{array}{l}\hat{b} \\
\dot{b} \\
\end{array}$ & 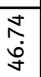 & $\begin{array}{l}\hat{n} \\
\stackrel{f}{f}\end{array}$ & $\begin{array}{l}\hat{0} \\
\dot{0} \\
\text { in }\end{array}$ & $\begin{array}{l}\widetilde{\sigma} \\
\dot{\forall}\end{array}$ & \begin{tabular}{l|}
\multirow{f}{*}{} \\
$\dot{f}$
\end{tabular} & $\begin{array}{l}\text { ?ै } \\
\text { in }\end{array}$ & $\begin{array}{l}\vec{m} \\
\stackrel{q}{q}\end{array}$ & $\begin{array}{l}\tilde{O} \\
\dot{m}\end{array}$ \\
\hline$\vec{x}$ & 両 & 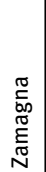 & $\stackrel{\infty}{\pi}_{\substack{\infty \\
0}}^{\infty}$ & $\frac{8}{5}$ & 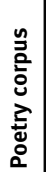 & 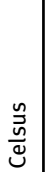 & 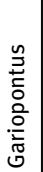 & $\begin{array}{l}\frac{n}{\bar{t}} \\
\frac{0}{0} \\
\overline{0} \\
\end{array}$ & 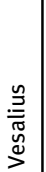 & 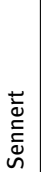 & $\begin{array}{l}\stackrel{\Xi}{\tilde{J}} \\
\infty \\
\bar{\S}\end{array}$ & 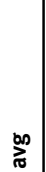 & जे & 름 & 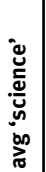 & 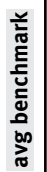 & 离 \\
\hline
\end{tabular}


Table 21: PoS values for the additional corpora as percentages. See table 12 for the general corpus.

\begin{tabular}{|c|c|c|c|c|c|c|c|}
\hline & $A D J$ & $A D V$ & CONJ & $\mathrm{N}$ & PREP & PRON & v \\
\hline Sallust & 10.87 & 8.92 & 9.72 & 29.85 & 7.42 & $\underline{10.23}$ & 22.99 \\
\hline Livy & $\underline{12.80}$ & 7.85 & $\underline{7.06}$ & $\underline{32.52}$ & 9.08 & $\underline{7.70}$ & 22.99 \\
\hline Suetonius & 12.11 & 8.91 & 10.10 & $\underline{30.51}$ & 8.67 & $\underline{7.13}$ & 22.56 \\
\hline Rufinus & $\underline{13.65}$ & 8.64 & $\underline{7.39}$ & $\underline{30.89}$ & 8.56 & $\underline{8.61}$ & 22.25 \\
\hline Orosius & 8.25 & 9.38 & 10.53 & 25.27 & 9.83 & 12.90 & 23.84 \\
\hline Gregory of Tours & 9.41 & 8.23 & $\underline{7.65}$ & 24.54 & 9.96 & 14.10 & $\underline{26.11}$ \\
\hline Notker Balbulus & $\underline{13.79}$ & 8.95 & 9.17 & 22.71 & 9.12 & 13.46 & 22.80 \\
\hline Liutprand of Cremona & $\underline{12.70}$ & 10.07 & $\underline{7.29}$ & 24.63 & 6.75 & 14.23 & 24.33 \\
\hline Adam of Bremen & $\underline{13.41}$ & 8.45 & $\underline{7.50}$ & 26.74 & 9.64 & 12.59 & 21.67 \\
\hline William of Malmesbury & 12.35 & 7.80 & $\underline{8.06}$ & $\underline{32.58}$ & 7.89 & $\underline{9.55}$ & 21.78 \\
\hline loh. de Plano Carpini & 8.80 & 9.10 & $\underline{12.80}$ & $\underline{20.46}$ & $\underline{11.36}$ & 14.72 & 22.76 \\
\hline Theodericus of Niem & $\underline{13.73}$ & 7.92 & $\underline{8.33}$ & 27.07 & 10.10 & 11.66 & 21.19 \\
\hline Valla & 10.05 & 9.73 & 10.31 & 26.42 & 8.99 & $\underline{10.50}$ & 24.00 \\
\hline Orlandini & $\underline{12.81}$ & 10.81 & $\underline{6.79}$ & 29.78 & 9.20 & $\underline{8.92}$ & 21.69 \\
\hline Thuanus & 10.70 & 9.66 & $\underline{7.56}$ & $\underline{29.91}$ & $\underline{11.04}$ & $\underline{8.04}$ & 23.09 \\
\hline avg & 11.70 & 8.87 & $\underline{8.61}$ & 27.61 & 8.68 & 11.05 & 23.48 \\
\hline stdev & 2.01 & 0.68 & 1.42 & 3.71 & 1.11 & 2.97 & 1.26 \\
\hline Charters & 11.90 & 7.86 & $\underline{11.61}$ & 26.24 & $\underline{11.10}$ & 12.90 & $\underline{18.38}$ \\
\hline avg 'science' (chap. 18) & 12.36 & 9.74 & 9.86 & 24.49 & 9.13 & 12.55 & 21.87 \\
\hline avg benchmark & 10.06 & 8.95 & 9.93 & 25.69 & 8.30 & 14.13 & 22.93 \\
\hline Lucretius & 12.00 & 10.42 & 9.84 & 26.62 & 6.35 & $\underline{10.39}$ & 24.39 \\
\hline Manilius & $\underline{14.48}$ & $\underline{4.88}$ & $\underline{7.42}$ & 33.94 & 6.68 & $\underline{8.10}$ & 24.50 \\
\hline Aetna & $\underline{16.07}$ & 7.95 & $\underline{7.93}$ & $\underline{30.20}$ & $\underline{3.90}$ & $\underline{8.29}$ & 25.67 \\
\hline Avienus & $\underline{15.71}$ & 9.89 & $\underline{6.32}$ & $\underline{32.30}$ & 6.18 & $\underline{8.88}$ & 20.72 \\
\hline Walahfrid & $\underline{17.36}$ & $\underline{7.32}$ & $\underline{6.48}$ & $\underline{34.52}$ & $\underline{3.66}$ & $\underline{7.77}$ & 22.89 \\
\hline Macer Floridus & 12.45 & $\underline{6.22}$ & $\underline{7.20}$ & 29.00 & 4.42 & $\underline{10.19}$ & $\underline{30.53}$ \\
\hline Palingenius & $\underline{15.08}$ & 10.21 & 9.47 & 25.70 & $\underline{3.52}$ & $\underline{11.28}$ & 24.76 \\
\hline Bruno & $\underline{16.37}$ & $\underline{7.36}$ & $\underline{8.56}$ & $\underline{30.09}$ & $\underline{5.88}$ & $\underline{9.35}$ & 22.37 \\
\hline Stay & $\underline{12.99}$ & 11.27 & $\underline{8.59}$ & 25.86 & 6.52 & $\underline{9.92}$ & 24.86 \\
\hline Zamagna & $\underline{17.19}$ & 9.68 & 8.68 & 28.41 & $\underline{5.68}$ & $\underline{7.72}$ & 22.64 \\
\hline
\end{tabular}


Table 21: (continued)

\begin{tabular}{l|r|r|r|r|r|r|r}
\hline & ADJ & ADV & CONJ & N & PREP & PRON & V \\
\hline avg & $\underline{14.97}$ & $\mathbf{8 . 5 2}$ & $\underline{\mathbf{8 . 0 5}}$ & 29.66 & $\underline{5.28}$ & $\underline{9.19}$ & 24.33 \\
\hline stdev & 1.93 & 2.08 & 1.19 & 3.18 & 1.26 & 1.23 & 2.63 \\
\hline Poetry corpus & $\underline{15.92}$ & $\underline{7.04}$ & $\underline{7.09}$ & $\underline{30.90}$ & $\underline{4.27}$ & $\underline{10.21}$ & 24.58 \\
\hline Celsus & 12.28 & $\underline{11.48}$ & 10.69 & 23.68 & 7.12 & 11.16 & 23.60 \\
\hline Gariopontus & 11.59 & 8.54 & $\underline{16.64}$ & 23.58 & 7.78 & $\underline{8.47}$ & 23.40 \\
\hline Bernardus de Gordonio & $\underline{15.92}$ & 10.37 & $\underline{16.25}$ & $\underline{20.22}$ & 10.59 & $\underline{6.46}$ & 20.18 \\
\hline Vesalius & $\underline{16.31}$ & $\underline{11.81}$ & $\underline{7.69}$ & 28.15 & 7.15 & $\underline{10.71}$ & $\underline{18.18}$ \\
\hline Sennert & $\underline{13.15}$ & $\underline{10.67}$ & $\underline{11.85}$ & 23.85 & 8.53 & $\underline{10.11}$ & 21.85 \\
\hline von Bene & $\underline{20.36}$ & 9.89 & $\underline{8.10}$ & 27.61 & 9.19 & $\underline{4.99}$ & 19.86 \\
\hline avg & $\underline{14.93}$ & 10.46 & $\underline{11.87}$ & $\mathbf{2 4 . 5 2}$ & $\mathbf{8 . 3 9}$ & $\underline{\mathbf{8 . 6 5}}$ & $\mathbf{2 1 . 1 8}$ \\
\hline stdev & 3.28 & 1.18 & $\mathbf{3 . 8 7}$ & 2.94 & 1.34 & 2.48 & 2.14 \\
\hline Pliny & $\underline{14.40}$ & $\underline{7.14}$ & $\underline{8.10}$ & $\underline{32.75}$ & $\mathbf{8 . 7 9}$ & $\underline{8.78}$ & 20.03 \\
\hline avg 'science' (chap.19) & 12.36 & $\mathbf{9 . 7 4}$ & $\mathbf{9 . 8 6}$ & $\mathbf{2 4 . 4 9}$ & $\mathbf{9 . 1 3}$ & 12.55 & $\mathbf{2 1 . 8 7}$ \\
\hline avg benchmark & $\mathbf{1 0 . 0 6}$ & $\mathbf{8 . 9 5}$ & $\mathbf{9 . 9 3}$ & $\mathbf{2 5 . 6 9}$ & $\mathbf{8 . 3 0}$ & $\mathbf{1 4 . 1 3}$ & $\mathbf{2 2 . 9 3}$ \\
\hline stdev & $\mathbf{0 . 8 7}$ & $\mathbf{0 . 5 3}$ & $\mathbf{0 . 4 3}$ & 1.40 & $\mathbf{0 . 7 7}$ & 1.12 & 1.03 \\
\hline
\end{tabular}

Again using PCA with the nine values that seemed distinctive for science, the historical texts do not form a very neat cluster and generally remain between the non-scientific benchmark samples and the poetry samples, but far from the science samples (fig. 44). This indicates that historiography used a rather less technical language than the other fields hitherto considered. Methodologically more sound historians cannot be differentiated from 'propagandists': the difference clearly lies in the content, not in the language. Suetonius is the furthest from the benchmark samples; some authors are close to the charters sample - especially Sallust, Rufinus, Gregory of Tours - indicating a plausible kinship between these two kinds of texts. After all, many historical writers use charters as sources. Iohannes de Plano Carpini's language differs conspicuously from the other authors.

The case is very different for scientific poetry (fig. 45): these texts cluster together much more neatly, indeed somewhere between other poetry and scientific prose texts (especially medicine) and far from the benchmark samples - quite as expected. Stylometric plots for these two samples produce similar results: scientific poetry groups neatly, historiography does not (plots not printed). 


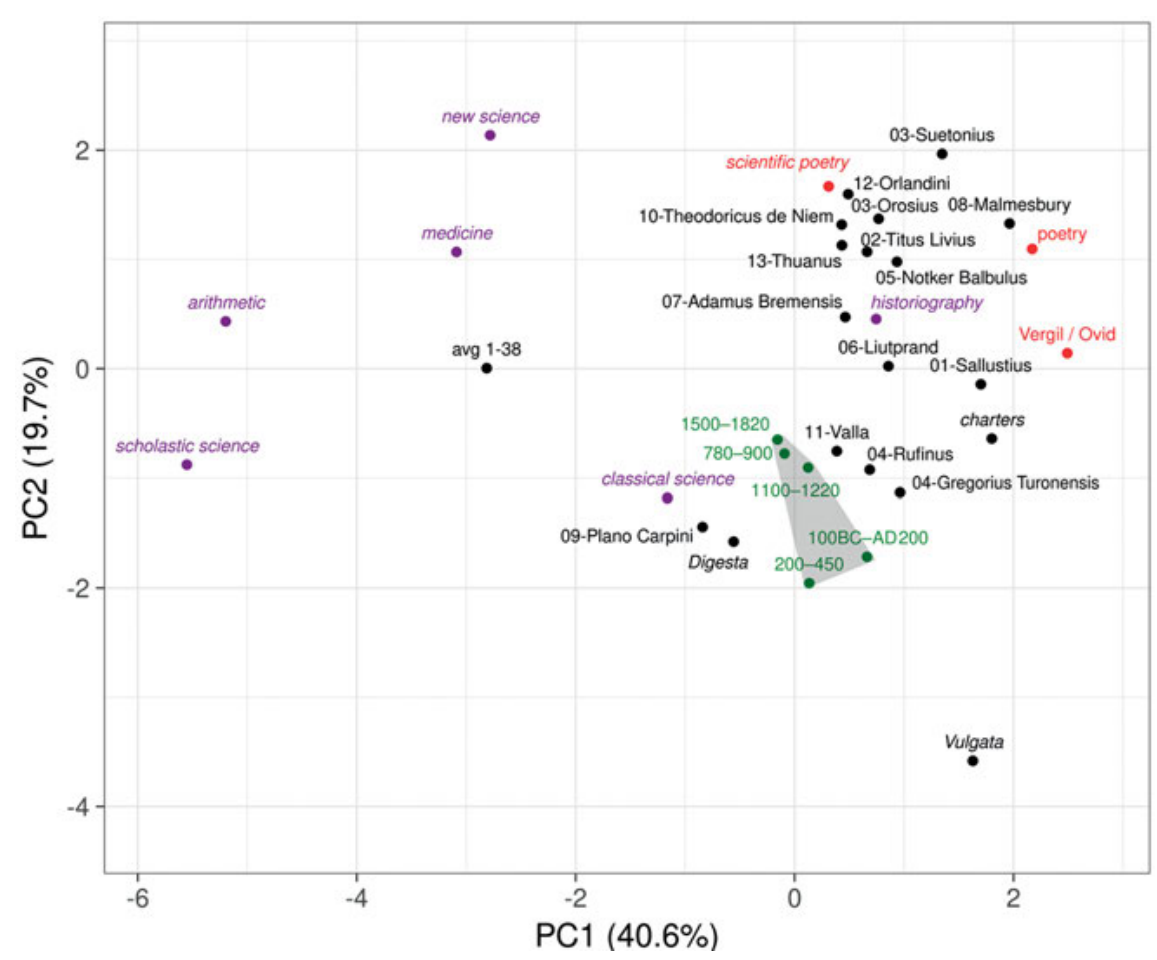

Fig. 44: Plot using the nine parameters from above (see fig. 38, except entropy) for the historiographical texts. The texts group around the benchmark samples.

\$6 For both these additional samples, it has become clear that the language differs strongly from the scientific texts studied so far. Table 22 summarises the conspicuous values for the corpora and most clear-cut groups of texts that have been studied. It becomes clear that the historiographical sample differs significantly even more so than the scientific poetry sample - from the clearly scientific samples (general sample, scholasticism, arithmetic, medicine, less clearly jurisprudence). Among the latter, it is the medical texts that differ most strongly; the other three samples agree in the parameters ESSE, ADJ-SUF, modifiers, and entropy (underlined). 1st SG, 3rd PAS, and PRON:POSS may be the most distinctive values for scientific texts (bold). The scholastic sample represents the most extreme type of scientific Latin, focusing on NOM, IND, and using suffixes and modifiers, apparently in emulation of Greek, while CONJ and PREP may be more used than bare clauses and cases in order to increase perspicuity - all in all, approaching an artificial logical language and language engineering. 


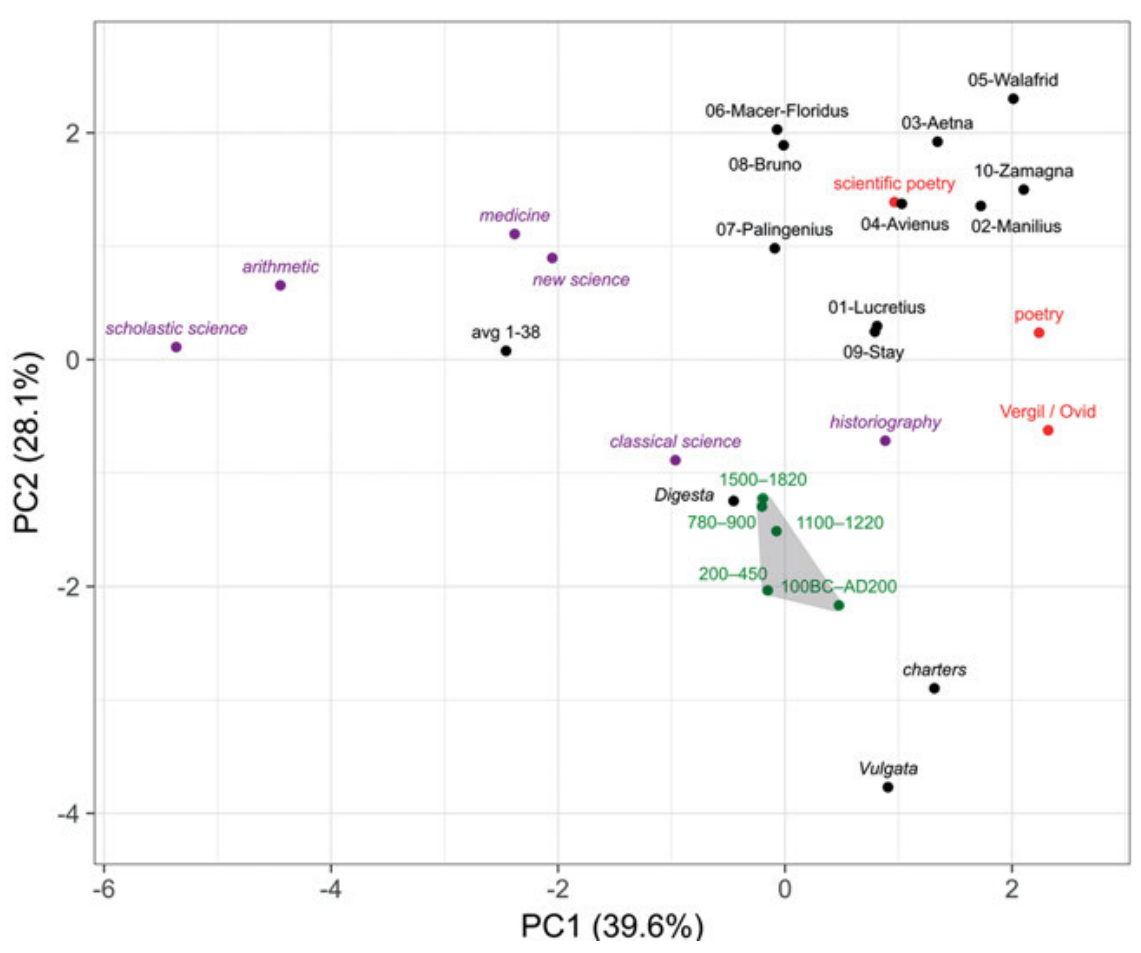

Fig. 45: Plot using the nine parameters from above (see fig. 39) for scientific poetry. The texts are clearly apart from the rest, including normal poetry.

Table 22: The table summarises the average values of the corpora studied in contrast to the prose benchmark samples. +++: more than 3 stdevs above the benchmark samples, + more than 1 stdev above; analogously for - and - - - . Parameters that are not included are, therefore, within 1 stdev of the benchmark average. Non-scientific poetry is shown as an out-group. Especially interesting parameters are marked.

\begin{tabular}{|c|c|c|c|c|}
\hline & +++ & + & - & --- \\
\hline $\begin{array}{l}\text { General sample from } \\
\text { chap. } 18 \text { ( } 40 \text { texts) }\end{array}$ & $\begin{array}{l}\text { NOM, ESSE, 3rd PAS, } \\
\underline{\text { ADJ-SUF }}\end{array}$ & $\begin{array}{l}\text { ADJ, ADV, PREP, N- } \\
\text { SUF, modif. }\end{array}$ & $\begin{array}{l}\text { N, PRON, V, ACC, 1st } \\
\text { SG, entropy }\end{array}$ & POSS \\
\hline Scholasticism ${ }^{23}$ & 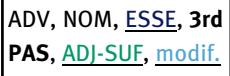 & $\begin{array}{l}\text { ADJ, CONJ, PREP, IND, } \\
\text { REL, N-SUF }\end{array}$ & $\begin{array}{l}\text { SUB, PTC, GEN, ABL/ } \\
\text { DAT, 1st SG }\end{array}$ & $\begin{array}{l}\text { N, ACC, POSS, ABL } \\
\text { ABS, entropy }\end{array}$ \\
\hline Arithmetic & 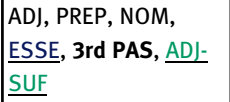 & CONJ, modif. & $\begin{array}{l}\text { PRON, V, INF, GEN, } \\
\text { ACC, ABL/DAT, 1st } \\
\text { SG, ABL ABS }\end{array}$ & N, POSS, entropy \\
\hline
\end{tabular}

23 Average from the texts in the general corpus by Albertus, Aquinas, Duns, and Ockham, who seem especially typical. 
Table 22: (continued)

\begin{tabular}{l|l|l|l|l}
\hline & +++ & + & - & --- \\
\hline Medicine & $\begin{array}{l}\text { ADJ, CONJ, 3rd PAS, } \\
\text { POSS, ADJ-SUF }\end{array}$ & $\begin{array}{l}\text { ADV, NOM, ESSE, ABL } \\
\text { ABS }\end{array}$ & $\begin{array}{l}\text { N, V, INF, GEN, ACC, } \\
\text { REL, 1st SG, N-SUF }\end{array}$ & PRON \\
\hline Jurisprudence $^{24}$ & ESSE, CONJ:S & $\begin{array}{l}\text { CONJ, V, SUB, INF, } \\
\text { ABL/DAT, 3rd PAS, N- } \\
\text { SUF }\end{array}$ & $\begin{array}{l}\text { ADJ, IND, PTC, GEN, } \\
\text { 1st SG, ADJ-SUF }\end{array}$ & POSS \\
\hline Historiography & PTC, ACC, ABL ABS & $\begin{array}{l}\text { ADJ, N, PREP, ACC, } \\
\text { ABL/DAT, entropy }\end{array}$ & $\begin{array}{l}\text { CONJ, PRON, IND, } \\
\text { GEN, CONJ:S, POSS, } \\
\text { 3rd PAS, N-SUF, } \\
\text { modif. }\end{array}$ & NOM, ESSE \\
\hline Scientific poetry & ADJ, PTC, ABL ABS & N, V, ACC, entropy & $\begin{array}{l}\text { PRON, SUB, GEN, } \\
\text { REL, CONJ:S 1st SG, } \\
\text { 3rd PAS, modif. }\end{array}$ & $\begin{array}{l}\text { CONJ, PREP, PRON, } \\
\text { ESSE, POSS, N-SUF }\end{array}$ \\
\hline Non-scientific poetry & $\begin{array}{l}\text { ADJ, N, ACC, ABL ABS, } \\
\text { entropy }\end{array}$ & V, PTC, NOM & $\begin{array}{l}\text { SUB, REL, ADJ-SUF } \\
\text { ADV, CONJ, PREP, }\end{array}$ & $\begin{array}{l}\text { PRON, GEN, ESSE, } \\
\text { CONJ:S, 3rd PAS, N- } \\
\text { SUF, modif. }\end{array}$ \\
\hline
\end{tabular}

\$7 As a summary of these corpus approaches, a final plot (fig. 46) shows all the used texts together, marking the disciplines or genres in different colours. The colours show that most groups cluster together quite closely, from left to right: arithmetic, scholastic authors (some early and late ones are not coloured), translations from Greek, medical writers, juridical texts, didactic poetry, historiography. Only the medical and juridical writers are quite scattered, and only the historiographical ones are close to the benchmark samples. Thus, historiography tends to remain close to 'normal' prose, indeed between it and the charters sample; scientific poetry is also quite apart from the other texts, between non-scientific poetry and scientific prose. Modern human-science authors (Vossius, Kircher, Kretschmann) seem to use a style between that of historiographers and the natural scientists of the Scientific Revolution, who are situated quite in the middle of the plot (Copernicus, Galileo, Newton). For Boethius and Isidore, there are two samples each, one from the general texts and one of an arithmetic text. It is interesting to note that the arithmetic ones group quite far away from the general ones, as expected within the arithmetic group. Ficino's translation and Iohannes de Plano Carpini behave unexpectedly for their groups.

24 Average of Gaius, Pauli sententiae, and Digesta. 


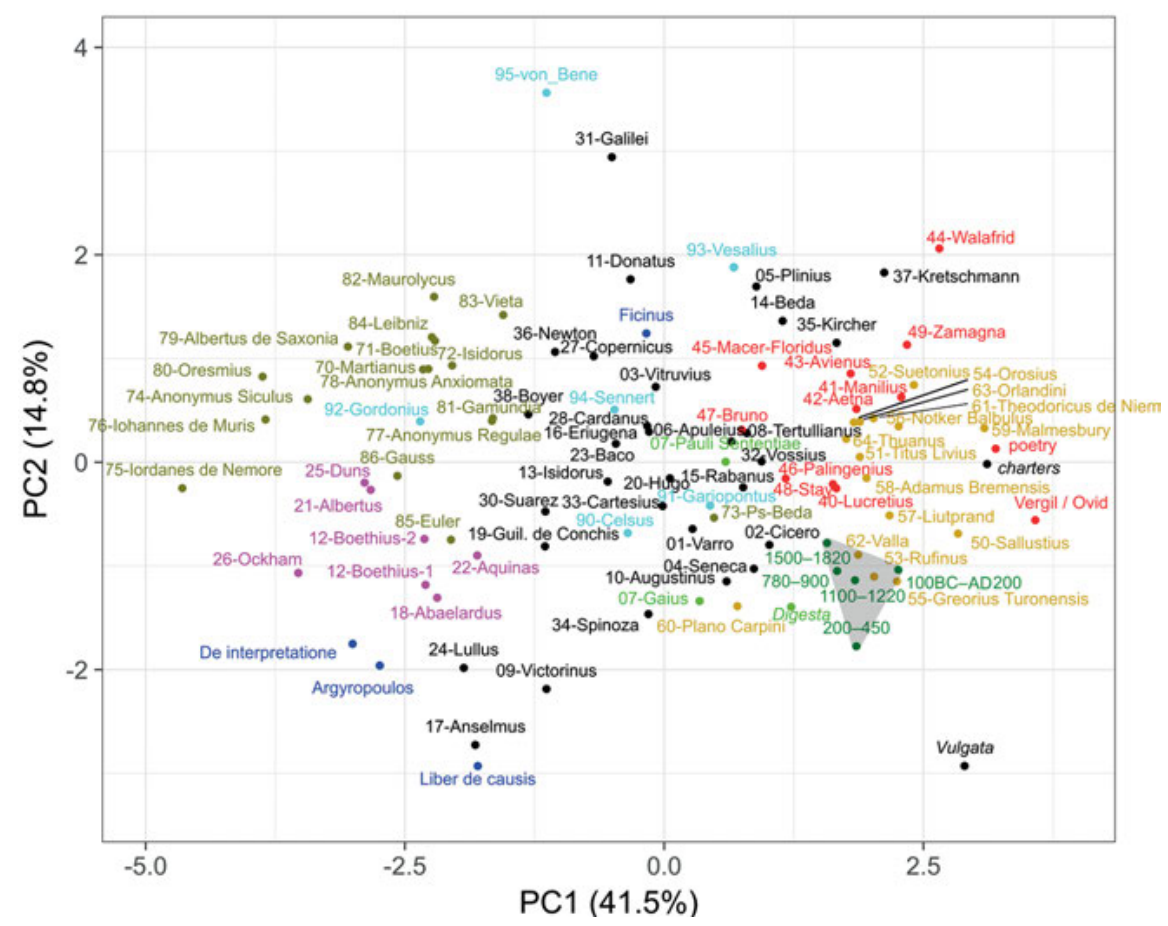

Fig. 46: A plot using the same nine parameters (see fig. 38), this time including all the texts considered (but no groups). The colours highlight disciplines and genres: benchmark (green), translations (blue), medicine (light blue), scientific poetry (red), historiography (yellow), arithmetic (brown), juridical (light green), and scholastic (pink).

\$8 Some general conclusions from the numerical research in this and the previous two chapters can now be drawn. The general corpus showed the existence of some quite neatly differentiable styles of Latin scientific prose (summarised in chap. 19). By means as simple as the PoS distribution, scholastic texts can be clearly separated from classicist or technical ones. The parameters that, it was hoped, would be significant for scientific writing (departing from what is known about scientific English and German) have led to mixed results for Latin: some of them differ strongly from other Latin, while others do not. The former are not always the same ones as those known to differ for scientific English. As in English scientific language, in Latin science PREP, 3rd PAS, and ESSE are indeed more common, but CONJ or GEN are not. The passive voice is more common in scientific Latin than otherwise, but not in such an extreme way as in contemporary scientific English. The 1st SG and possessive pronouns are also less common. Some features typical for scientific English do not exist in the same way in Latin, such as the -ing-form. Unexpectedly, possible Latin counterparts such as participles, infi- 
nitives, and the ablativus absolutus are less common in scientific writing than otherwise; even the relative pronoun (REL) is only marginally more common in the general science corpus than in the benchmark samples. Also surprisingly, subordination (CONJ:S) does not seem to be significantly more common in scientific Latin texts. The greater frequency of suffixes is a feature not previously studied for scientific English; ADJ suffixes, in particular, are conspicuous in scientific Latin. Other features with no known corresponding behaviour in scientific English are higher numbers of typically Greek sentence-modifying particles and lower entropy. The lower entropy may indicate a smaller density of information (which would be unexpected) and/or more monotone vocabulary and less variatio (which would be expected).

In some fields, a rather coherent type of language could be found: arithmetic, historiography, scientific poetry, scholasticism, and to a lesser extent medicine and jurisprudence. The arithmetic corpus has revealed an especially monolithic style these authors have used for over more than a millennium; it proved possible to link it to Euclid. Of course, this 'hard' science is especially little prone to rhetoric and linguistic variation. Numerals are necessarily among the most common words, and this science is based on proofs, which tend to be linguistically monotonous. Scientific texts translated from Greek tend to have more modifier particles (unsurprisingly) but also more CONJ and PREP, more NOM and IND, and lower entropy. It would be interesting to study which of these factors represent general differences between Greek and Latin. Some typically mediaeval Latin features were also found in passing (in the mediaeval benchmarks in contrast to those before and after): lower numbers of INF and, less clearly, lower numbers of ADJ and lower entropy. The kind of scientific Latin that departed the most from normal prose was clearly scholastic Latin. This most far-reaching approach to Latin language engineering was reined in by humanist disdain for such 'ugly' Latin. The two later typical forms of Latin science, that of the natural-science revolution and that of early modern human sciences and scholarship, deviated less from 'normal' Latin. Apart from this development, clear diachronic change was rarely observed; only in the arithmetic sample texts, pre-Euclid-translation texts and modern authors (Leibniz, Euler, Gauß) were set apart, at least in the plots based on the most common vocabulary (fig. 43). It may be pointed out that in general, Latin scientific language does not differ from non-technical language as much as is the case with some other languages. For instance, although there are slightly different ratios of cases used in some genres (as was seen above), there are no special uses of cases as there are in scientific Sanskrit. ${ }^{25}$

25 This very elaborate system is described by Jacobi (1970); see also Staal (1995). 
It should be added that (as already indicated above) the quality of the data is not as good as it could be. Computational linguistics is currently in a phase of rapid development, and tools missing for Latin may soon be available (e.g. through Marco Passarotti's LiLa project). Tagging, the amount of data, and methodology will be much better in a mere ten years' time. It remains to be seen to what extent the results presented here will stand the test of time. After these first ever corpusbased studies of the Latin used by scholars and scientists, for the remainder of this book the scope will be widened and scientific Latin will be compared to other languages of science. We return first of all to the question of scientific vocabulary. 